\title{
Asynchronous glutamate exocytosis is enhanced in low release probability synapses and is widely dispersed across the active zone.
}

\section{Philipe Mendonca}

Federal University of Minas Gerais

\section{Erica Tagliatti}

UCL Queen Square Institute of Neurology

\section{Helen Langley}

UCL Queen Square Institute of Neurology, University College London

\section{Dimitrios Kotzadimitriou}

UCL Queen Square Institute of Neurology, University College London

\section{Criseida Zamora-Chimal}

University of Warwick

\section{Yulia Timofeeva}

University of Warwick

Kirill Volynski ( $\square$ k.volynski@ucl.ac.uk)

UCL Queen Square Institute of Neurology, University College London

\section{Article}

Keywords: neurotransmitters, neuronal network, synapses

Posted Date: May 25th, 2021

DOl: https://doi.org/10.21203/rs.3.rs-537334/v1

License: (c) (i) This work is licensed under a Creative Commons Attribution 4.0 International License. Read Full License

Version of Record: A version of this preprint was published at Nature Communications on June 17th, 2022. See the published version at https://doi.org/10.1038/s41467-022-31070-4. 


\title{
Title: Asynchronous glutamate exocytosis is enhanced in low release probability synapses and is widely dispersed across the active zone.
}

\section{Authors:}

Philipe R. F. Mendonça ${ }^{1,2 *}$, Erica Tagliatti ${ }^{1}$, Helen Langley ${ }^{1}$, Dimitrios Kotzadimitriou ${ }^{1}$, Criseida G. Zamora-Chimal ${ }^{3}$, Yulia Timofeeva ${ }^{1,3 *}$ and Kirill E. Volynski ${ }^{1 *}$

\author{
Affiliations: \\ ${ }^{1}$ University College London Institute of Neurology; London, UK; \\ ${ }^{2}$ Department of Physiology and Biophysics, Federal University of Minas Gerais; Brazil; \\ ${ }^{3}$ Department of Computer Science, University of Warwick; Coventry, UK; \\ *Corresponding authors.k.volynski@ucl.ac.uk,p-mendonca@ufmg.br and \\ y.timofeeva@warwick.ac.uk
}

\begin{abstract}
The balance between fast synchronous and delayed asynchronous release of neurotransmitters has a major role in defining computational properties of neuronal synapses and regulation of neuronal network activity. However, how it is tuned at the single synapse level remains poorly understood. Here, using the fluorescent glutamate sensor SF-iGluSnFR, we image quantal vesicular release in tens to hundreds of individual synaptic outputs from single pyramidal cells with 4 millisecond temporal and $75 \mathrm{~nm}$ spatial resolution. We find that the ratio between synchronous and asynchronous synaptic vesicle exocytosis varies extensively among synapses supplied by the same axon, and that and that synchronicity of release is reduced at low release probability synapses. We further demonstrate that asynchronous exocytosis sites are more widely distributed within the release area than synchronous sites. Together, our results reveal a universal relationship between the two major functional properties of synapses - the timing and the probability of neurotransmitter release.
\end{abstract}

\section{Introduction}

Synaptic transmission provides the basis for neuronal communication. When an actionpotential propagates through the axonal arbour, it activates voltage-gated $\mathrm{Ca}^{2+}$ channels (VGCCs) located in the vicinity of release-ready synaptic vesicles docked at the presynaptic active zone ${ }^{1} . \mathrm{Ca}^{2+}$ ions enter the presynaptic terminal and activate the vesicular $\mathrm{Ca}^{2+}$ sensor Synaptotagmin 1 (Syt1, or its isoforms Syt2 and Syt9), thus triggering exocytosis of synaptic vesicles filled with neurotransmitter molecules. Neurotransmitter diffuses across the synaptic cleft, binds postsynaptic receptors and evokes further electrical or chemical signalling in the postsynaptic target cell. This whole process occurs on a time scale of a few milliseconds. Recent data demonstrate that such speed and precision are in large part achieved via the 
formation of nanocomplexes that include presynaptic VGCCs, vesicles belonging to a readily releasable pool (RRP) and postsynaptic neurotransmitter receptors ${ }^{2-4}$.

In addition to fast, synchronous release, which keeps pace with action potentials, many synapses also exhibit delayed asynchronous release that persists for tens to hundreds of milliseconds ${ }^{1,5}$. Asynchronous release is potentiated during repetitive presynaptic firing and is triggered via activation of multiple sensors with both low (e.g. Syt1) and high (e.g. Syt7) $\mathrm{Ca}^{2+}$ affinity $^{6}$. Accumulating evidence demonstrates that the balance between synchronous and asynchronous release plays an important role in coordinating activity within neuronal networks, for example, by increasing the probability of postsynaptic cell firing and/or modulating action potential precision ${ }^{7-10}$. It is well established that asynchronous release levels vary among different types of neurons ${ }^{1}, 10,11$. Interestingly, recent data show that the ratio between asynchronous and synchronous release can also be differentially regulated among presynaptic boutons supplied by the same axon and depends on the identity of the postsynaptic cell, which contributes to target cell-specific communication in the brain ${ }^{7,8}$.

The mechanisms that control the relative contributions of synchronous and asynchronous release at the level of single synapses are however poorly understood. Variability in asynchronous release among different neuronal types has been attributed to differences in synaptic morphology (e.g. the coupling distance between RRP vesicles and VGCCs $)^{10}$ and to cell-type specific expression of components of the vesicular release machinery $\left(e . g\right.$. the $\mathrm{Ca}^{2+}$ sensors Syt1 and Syt7) ${ }^{11}$. Whether the same mechanisms contribute to the regulation of release modes among presynaptic boutons located on the same axon remains unclear. It is also unknown whether synchronous and asynchronous release occur from the same or different pools of synaptic vesicles. Recent functional electron microscopy analysis combined with fast high-pressure freezing have helped to visualise loci of either synchronous or asynchronous release events within a single active zone ${ }^{4,12}$. Application of this approach to synaptic populations indicates that synchronous and asynchronous release events tend to occur in different sub-domains of the active zone. However, how synchronous and asynchronous release sites are located with respect to each other within the same active zone has not yet been established.

\section{Results}

Imaging of synchronous and asynchronous vesicular release across axonal arbour To address these questions, we developed a novel imaging technique and data analysis framework, which allowed us to directly investigate the relationship between synchronous and asynchronous glutamate release both at the level of individual active zones and across large populations of synaptic outputs from a single pyramidal neuron. Our approach (Fig. 1) is based on expression of the fluorescent glutamate reporter SF-iGluSnFR on the axonal membrane, which allows detection of glutamate release from individual synaptic vesicles with millisecond resolution ${ }^{13-16}$. We sparsely transfected neocortical neurons in culture with SF-iGluSnFR and established a whole-cell voltage-clamp recording in a neuron with pyramidal morphology expressing the sensor (Fig. 1A). We next imaged action potential-evoked glutamate release by monitoring changes in SF-iGluSnFR fluorescence (at a rate of $4 \mathrm{~ms} /$ frame) in tens to hundreds of individual presynaptic boutons supplied by the axon of the selected neuron, in response to a $5 \mathrm{~Hz}$ train of 51 action potentials triggered by brief somatic voltage steps (Fig. 1B-E, Methods). 
By applying a set of spatial-temporal filters and calculating the maximal projection of the resulting image stack, we could identify all active boutons irrespective of their release probability, as long as they released at least one vesicle during the train (Fig. 1D and Movie S1). Evoked synaptic SF-iGluSnFR responses had a stereotypical waveform with a quasiinstantaneous rising phase that was followed by a slower exponential decay, corresponding to glutamate unbinding from SF-iGluSnFR molecules $\left(\tau \sim 68 \mathrm{~ms}\right.$, Fig. $1 \mathrm{E}$ and Fig. S1) ${ }^{14}$. We applied a deconvolution procedure (using the averaged SF-iGluSnFR response waveform) to determine the precise timings and the amplitudes of release events (Fig. 1E, Figs. S1-S3 and Methods). The histograms of deconvolved event amplitudes at individual boutons typically showed between one and four discernible quantal peaks, consistent with the exocytosis of one or more vesicles of glutamate (Fig. 1E and Figs. S4 and S5) ${ }^{13,15,17}$. By fitting the histogram with a sum of Gaussian functions we estimated the amplitude of the SF-iGluSnFR signal corresponding to the release of a single quantum ( $q$, Fig. 1E and Methods). Critically, application of this quantal analysis allowed us to estimate the number of vesicles released during each event and therefore directly compare vesicular exocytosis among different synapses. We note that the absolute values of the normalised evoked SF-iGluSnFR signals ( $\left.\Delta F / F_{0}\right)$ depend not only on the number of exocytosed vesicles but also on the presynaptic bouton size and geometry, which significantly vary among synapses (Fig. 1E, Figs.S4 and S5). Therefore, the use of $\Delta F / F_{0}$ is not optimal for quantitative comparison of vesicular release parameters.

To define a time threshold for separation of synchronous and asynchronous release events, we calculated the average time course of vesicular exocytosis following an action potential across all recorded cells. This allowed us to compare the imaging data at single synapses to previous electrophysiological recordings from synaptic populations (e.g. refs. ${ }^{5,10,18}$ ). In agreement with the electrophysiological data, the vesicular release time-course determined with SF-iGluSnFR followed a well-defined biphasic shape (Fig.1E). We note that although synchronous release is expected to occur within 1-2 ms after the presynaptic spike ${ }^{1}$, due to the finite speed of action potential propagation, the onset of synchronous release is delayed by $\sim 2-6 \mathrm{~ms}$ with respect to the somatic action potential (as boutons are located several hundred micrometres away from the soma, Fig. 1A) ${ }^{19}$. This blurs the distribution of detected synchronous release events and therefore we have used $10 \mathrm{~ms}$ as a threshold for the separation of synchronous and asynchronous exocytosis components.

\section{Asynchronous release is elevated in low release probability synapses}

We next tested how synchronous and asynchronous release events are distributed among synaptic outputs of individual pyramidal neurons. By using the defined $10 \mathrm{~ms}$ threshold we classified events as synchronous or asynchronous (blue and red colour codes respectively in Fig. 1E and subsequent figures) and calculated the total release rate ( $n_{T}$, average number of vesicles released per action potential) and the fraction of asynchronous release events $\left(n_{A} / n_{T}\right)$ for each bouton. In line with previous studies (e.g. refs. $\left.{ }^{15,20}\right), n_{T}$ (which is directly related to the overall release probability $P_{r e l}$ ) varied widely among presynaptic boutons supplied by the same axon. Unexpectedly, we found an inverse relationship between $n_{T}$ and 
the asynchronous release fraction, which was elevated at boutons with low release probability (Fig. 2A and examples in Fig. S4). To quantify this phenomenon, we divided boutons in each recorded cell into two groups with low and high $P_{r e l}$ (using the median value for $n_{T}$ ). We found that asynchronous release fraction was on average $\sim 1.5$-fold higher in low $P_{r e l}$ than in high $P_{\text {rel }}$ boutons (Fig. 2A, $n_{A} / n_{T}=0.31 \pm 0.04$ and $n_{A} / n_{T}=0.20 \pm 0.02$ respectively).

It has been demonstrated that the variability in expression levels of the $\mathrm{Ca}^{2+}$ sensor for synchronous vesicular release Syt1 has a major role in the regulation of the balance between synchronous and asynchronous release among different types of neurons ${ }^{11}$. To test for a possible role of Syt1 in controlling the heterogeneity of vesicular release modes among synaptic outputs of a single neuron we repeated our experiments in neuronal cultures from $\mathrm{Syt}^{-/-}$mice. As expected, deletion of Syt1 resulted in $\sim 4-5$-fold decrease of synchronous and correspondingly $\sim 4-5$-fold increase of asynchronous vesicular exocytosis (Fig. 1F, G and Figs $\mathrm{S} 4$ and S5 ${ }^{18}$. However, in spite of the overall increase of asynchronous release, we still observed the reciprocal relationship between asyncrnonous release fraction and $P_{r e l}$ (Fig. 2B, $n_{A} / n_{T}=0.82 \pm 0.02$ in low $P_{r e l}$ and $n_{A} / n_{T}=0.74 \pm 0.03$ in high $P_{r e l}$ boutons). This indicates that the balance between different release modes at synapses supplied by a single neuron is at least in part determined by mechanisms distinct from the possible variation in the expression levels of Syt1.

\section{Relationship between short-term facilitation and synchronous/asynchronous release balance}

Typically, synapses with low $P_{r e l}$ display short-term facilitation of synchronous release during repetitive stimulation. Our finding that asynchronous release fraction is higher at low $P_{r e l}$ synapses suggests that the balance between different release modes could be universally linked to the type of short-term plasticity expressed in the same presynaptic terminals. To test this hypothesis, we imaged vesicular release in response to 10 pairs of action potentials delivered at $20 \mathrm{~Hz}$ (50 ms inter-spike interval, $10 \mathrm{sec}$ between paired pulses) and calculated in each recorded bouton the paired-pulse ratio ( $P P R$ ), the asynchronous release fraction $n_{A} / n_{T}$ and the total release rate $n_{T}$. We then compared the distributions of these major functional presynaptic properties among synaptic outputs of individual neurons and found that asynchronous release fraction was indeed $\sim 2$-fold higher in facilitating than in depressing synapses (Fig. 2C, $n_{A} / n_{T}$ $=0.058 \pm 0.007$ and $n_{A} / n_{T}=0.034 \pm 0.005$ respectively; note that as asynchronous release is potentiated during trains of action potentials ${ }^{1}$ the overall fraction of asynchronous release measured after two pulses was several fold lower than the fraction of asynchronous release during $5 \mathrm{~Hz}$ stimulation).

\section{Super-resolution localisation of synchronous and asynchronous exocytosis sites}

We next asked what are the spatial distributions of synchronous and asynchronous events within individual active zones. During vesicular exocytosis glutamate can be assumed to be released from a point source. Therefore, fusion of a single vesicle is expected to generate a bell-shaped SF-iGluSnFR fluorescence profile centred at the exocytosis site, which can be 
fitted using a 2D Gaussian function to determine the location of vesicular exocytosis with subpixel precision ${ }^{16}$. We applied this approach to directly compare locations of synchronous and asynchronous release events within the same presynaptic bouton. By using quantal analysis, we first selected single-vesicle fusion events (Fig. 3A). We next generated the corresponding 'Event images' for sub-pixel localisation of release sites, by applying pixel-by-pixel temporal deconvolution of the unitary SF-iGluSnFR response to the original image time series (Fig. 3B and Methods). The deconvolution procedure allowed us to specifically extract and amplify the spatial component SF-iGluSnFR fluorescence signal associated with the vesicular exocytosis event. This increased the signal-to-noise ratio and enabled us to localise positions of individual release events with approximately $75 \mathrm{~nm}$ precision (50 - $100 \mathrm{~nm}$ range, Fig. 3C, Fig. S6 and Movie S2). In $95 \%$ of boutons, vesicular exocytosis sites were clustered within a single compact area $\left(S=0.09 \pm 0.01 \mu \mathrm{m}^{2}\right.$ in wild type and $S=0.10 \pm 0.01 \mu \mathrm{m}^{2}$ at Syt1 KO synapses, Fig. 3D,E and Fig. S7), whilst the remaining 5\% of boutons contained two separate release areas. We note that due to exclusion of multivesicular release events and single vesicle events that could not be localised with sufficient precision (i.e. within the specified $100 \mathrm{~nm}$ threshold, Methods and Fig. S6) we could only determine locations of approximately $50 \%$ of all events. Furthermore, the imaged boutons were randomly tilted with respect to the microscope axes. Therefore the release area determined with SF-iGluSnFR on the 2D image projection represents a lower limit of the corresponding active zone area ${ }^{21}$. Indeed, the average active zone size determined using 3D cryo-electron microscopy was $\sim 1$.35-fold larger $\left(\sim 0.12 \mu \mathrm{m}^{2}\right)$ than obtained with our approach. The release area size varied widely among presynaptic boutons and, as expected, correlated with the total number of vesicles released per action potential $\left(n_{T}\right)$ both in wild type and in Syt $1^{-/-}$synapses (Fig. 3E).

\section{Asynchronous events are more widely distributed across the presynaptic release area}

To compare the relative locations of synchronous and asynchronous exocytosis sites, we applied hierarchical cluster analysis (Fig. 3F and Methods). In line with previous studies ${ }^{2,21}$, locations of individual release events could be grouped into small clusters ( $<100 \mathrm{~nm}$ diameter), which likely correspond to distinct release sites that are reused during repetitive stimulation. We found that among clusters that had at least two events, $\sim 49 \%$ of clusters in wild type and $\sim 43 \%$ of clusters in Syt1 $^{-/}$neurons contained both synchronous and asynchronous events. This argues that locations of synchronous and asynchronous events overlap and that they can occur from the same sites. However, in wild type neurons, the fraction of synchronous events progressively increased with the number of events in the cluster. Furthermore, the area covered by synchronous events had a shallower dependency on the number of events (slope $0.003 \mu \mathrm{m}^{2}$ / event) than the area covered by asynchronous events (slope $0.011 \mu \mathrm{m}^{2} /$ event). Together these findings suggest that synchronous release is confined to narrower nanodomains within the active zone. To test this at the level of individual synapses we compared each bouton against reshuffled versions of itself (Fig. 3G, H). In each simulation we maintained the number of synchronous and asynchronous events detected in each bouton but randomly scrambled their locations. We next calculated the average areas covered by each release mode (among all simulations) and used these to normalise the experimental values. Our rational was as follows: normalised value greater than 1 indicates that real events have a sparser distribution than the 
random counterpart, whilst normalised value smaller than 1 indicates that the real events have a more compact distribution. The re-shuffling analysis showed that the normalised area was indeed greater for asynchronous than for synchronous release (Fig. 3H, I). We therefore conclude that asynchronous events in wild type synapses are more widely dispersed across the release area than synchronous events. In contrast, the overall prevalence of asynchronous release in $\mathrm{Syt1}^{-/-}$neurons, largely occluded the difference between spatial distributions of synchronous and asynchronous exocytosis sites, albeit more compact clustering of the remaining synchronous release events was still detected with the re-shuffling analysis (Fig. 3I).

\section{Discussion}

Which mechanisms could explain the differential regulation of synchronous and asynchronous exocytosis at inter- and intrasynaptic levels? Our finding that locations of synchronous and asynchronous events overlap to a large extent, argues that both release modes can occur from the same pool of vesicles. On the other hand, a wider distribution of asynchronous release loci indicates that the probability that a given vesicle will be released synchronously or asynchronously depends on its location within the active zone.

A simple model that can potentially explain our findings, is that the observed variability of release properties could be a consequence of heterogeneity in coupling distances between VGCC and RRP vesicles. The synapse-specific tuning of the coupling distance has been demonstrated in synaptic outputs of pyramidal cells in the neocortex and has been implicated in the target cell-specific regulation of release probability and short-term plasticity ${ }^{22}$. The probability of synchronous release steeply depends on the coupling distance between releaseready vesicles and VGCCs, because it is triggered by transient $\mathrm{Ca}^{2+}$-nano/microdomains with a steep spatial gradient. In contrast, asynchronous release is not expected to depend on the coupling distance, as it is triggered by a more global ('residual') increase in the presynaptic $\mathrm{Ca}^{2+}$ concentration (Fig. 4A) ${ }^{1}$. Therefore, it follows that an increase of coupling distance should lead (i) to a decrease of the overall release probability with concurrent increase of asynchronous release fraction and (ii) to a wider distribution of vesicular exocytosis within the active zone. To test whether our results are consistent with this hypothesis we performed experimentally constrained modelling of presynaptic $\mathrm{Ca}^{2+}$ dynamics and activation of vesicular fusion (Fig. 4B-G and Methods). The model was based on the recent functional electron microscopy and single-molecule localisation data showing that presynaptic VGCCs, synchronous release sites and postsynaptic AMPA receptors colocalise within $20-30 \mathrm{~nm}$ distance and that AMPA receptor clusters preferentially localise to the periphery of the postsynaptic density/active zone $^{2-4,12}$. The model reproduced both a greater asynchronous release fraction at low release probability boutons (Fig. 4E) and a wider distribution of asynchronous release loci within the release area (Fig. 4F), thus arguing that synapse specific adjustment of coupling distances between VGCCs and vesicular release sites could be a major factor that accounts for the differential regulation of synchronous and asynchronous release.

At first sight, our findings contradict the electron microscopy studies, which demonstrated that asynchronous release loci are more biased towards the centre of the active zone $e^{4,12}$. Synchronous release events are expected to concentrate around VGCC clusters, which are predominantly located at the periphery of the active zone ${ }^{2-4}$ (Fig. 4C). Then it follows that asynchronous events, which are located further away from the VGCC clusters, can indeed be 
positioned closer to the active zone centre but be more widely distributed across the release area than synchronous events (as supported by our results).

Accumulating data demonstrate that the coupling distance between VGCCs and release-ready vesicles is dynamically regulated by presynaptic scaffold proteins (in particular RIM and RBP2) $)^{23,24}$ and it remains to be established whether and how this signalling pathway is regulated by the identity of the postsynaptic target cell. It also remains to be determined whether the balance between different release modes in synapses supplied by the same axon is further tuned by the adjustment of the local abundance of different synaptotagmin isoforms. In summary, our results reveal a general principle that relates the two major functional properties of small central glutamatergic synapses - the release probability and the synchronicity of vesicular release. We demonstrate that the ratio between synchronous and asynchronous synaptic vesicle exocytosis varies extensively among presynaptic boutons supplied by the same axon, and that asynchronous release fraction is elevated in parallel with short-term facilitation at synapses with low release probability. Such fine-tuning of different release modes, together with target cell-specific adjustment of release probability and shortterm plasticity $7,22,25,26$, has the potential to provide vast flexibility for synaptic computations in neural circuits composed of different cell types.

\section{Materials and Methods}

\section{Neuronal cultures and SF-iGluSnFR expression}

Experiments conformed to the Animals (Scientific Procedures) Act 1986, and were approved by the UK Home Office. Primary cortical neurons were produced from either wild type (C57BL/6J; Charles River) or Syt1 ${ }^{-/}$(B6; 129S-Syt1tm1Sud/J; The Jackson Laboratory) postnatal day 0 mouse pups of both sexes and cultured in Neurobasal A/B27-based medium (Thermo Fisher Scientific). The cortices were dissected and dissociated by enzymatic digestion in $0.25 \%$ trypsin for $10 \mathrm{~min}$ at $37^{\circ} \mathrm{C}$ and then triturated using a standard p1000 micropipette. Neurons were plated on poly-L-lysine-treated 19-mm glass coverslips $(1 \mathrm{mg} / \mathrm{mL}$; SigmaAldrich) at a density of $\sim 100,000$ cells per coverslip. At 5 days in vitro ( 5 DIV) neurons were transfected with pAAV.hSynap.SF-iGluSnFR.A184V plasmid ${ }^{14}$ (addgene Plasmid \#106174) using Neuromag reagent (KC30800; OZ Biosciences). The transfection resulted in sparse expression of the iGluSnFR probe in a small subpopulation of neurons $(\sim 3 \%)$, which allowed us to select individual cells for imaging. Experiments were performed between 16 and 21 DIV.

\section{SF-iGluSnFR imaging of glutamate release}

\section{Experimental set up}

SF-iGluSnFR fluorescence imaging experiments were performed on an inverted Olympus IX71 microscope equipped with a Prime95B back illuminated CMOS camera (Teledyne Photometrics) using a $60 \mathrm{x}$ oil-immersion objective $(1.35 \mathrm{NA})$ with resulting image pixel size $183.3 \mathrm{~nm}$. SF-iGluSnFR fluorescence was recorded using a 470-nm excitation light-emitting diode (OptoLED Light Source, Cairn Research) and a 500-550 band-pass emission filter. Image acquisition was performed using $\mu$ Manager software ${ }^{27}$.

Experiments were conducted in a custom-made open laminar flow perfusion chamber (volume $0.35 \mathrm{ml}$, perfusion rate $\sim 1 \mathrm{ml} / \mathrm{min}$ ) at $23-25^{\circ} \mathrm{C}$. The imaging extracellular solution contained (in mM): $125 \mathrm{NaCl}, 26 \mathrm{NHCO}_{3}, 12$ Glucose, $1.25 \mathrm{NaH}_{2} \mathrm{PO}_{4}, 2.5 \mathrm{KCl}, 2 \mathrm{CaCl}_{2}, 1.3 \mathrm{MgCl}_{2}$ 
(bubbled with $95 \% \mathrm{O}_{2}$ and $5 \% \mathrm{CO}_{2}, \mathrm{pH} 7.4$ ). To ensure that recorded SF-iGluSnFR responses originate only from the stimulated axon, we suppressed recurrent activity in the neuronal network by blocking postsynaptic ionotropic glutamate and GABA receptors with (in $\mu \mathrm{M}) 50$ DL-AP5 (Abcam), 10 NBQX (Abcam), and 50 Picrotoxin (Tocris Bioscience).

\section{Electrophysiology}

A putative pyramidal-like neuron expressing the SF-iGluSnFR probe that did not contain any other transfected cells in its vicinity was selected for imaging. A whole-cell voltage clamp recording was established in the selected cell using a fire-polished borosilicate pipette (4-7 $\mathrm{M} \Omega$, Warner Instruments) and Axon Multiclamp 700B amplifier (Molecular Devices). The intracellular pipette solution contained (in $\mathrm{mM}$ ): $105 \mathrm{~K}^{+}$Gluconate, $30 \mathrm{KCl}, 10 \mathrm{HEPES}, 10$ Phosphocreatine-Na2, 4 ATP-Mg, 0.3 GTP-NaH 20,1 EGTA ( $\mathrm{pH}=7.3$, balanced with KOH). The Multiclamp commander software was used to measure the series resistance $(\sim 20 \mathrm{M} \Omega)$, which was compensated at $\sim 30 \%$. Signal was acquired at $20 \mathrm{kHz}$ (4-kHz Bessel-filtering) using a National Instrument board NI USB-6221 controlled with WinWCP software (created and provided by John Dempster, University of Strathclyde). A liquid junction potential of $-10 \mathrm{mV}$ was subtracted from the measurements post hoc. The recorded neuron was held at $-70 \mathrm{mV}$ and action potentials were evoked using brief voltage steps $(5 \mathrm{~ms})$ from $-70 \mathrm{mV}$ to $-10 \mathrm{mV}$, producing stereotypical 'escape' currents.

\section{Fluorescence imaging}

After establishing a patch-clamp recording, evoked synaptic SF-iGluSnFR responses were imaged in $259 \times 23 \mu \mathrm{m}(1412 \times 125$ pixels $)$ ROIs located within the axonal arbour $200-1000$ $\mu \mathrm{m}$ away from the soma. Images were acquired at $250 \mathrm{~Hz}$ sampling rate (4 ms/frame). The exposure time of each frame was captured with the data acquisition board. This allowed us to align the timing of captured frames to the electrophysiological recording, and therefore to determine the time between each vesicular release event and the preceding to action potential. For each neuron between 1 to 4 different ROIs were imaged with approximately 5 minutes interval between the trials. The stimulation protocols consisted either of 51 action potentials delivered at $5 \mathrm{~Hz}$, or of 10 pairs of action potentials delivered at $20 \mathrm{~Hz}(50 \mathrm{~ms}$ inter-spike interval) with 10 seconds between individual paired pulse sweeps.

\section{Image analysis}

Image analysis was performed offline using Image $(\mathrm{NIH})^{28}$ and MATLAB (MathWorks) custom-developed scripts.

\section{Identification of active boutons}

In order to detect all active presynaptic boutons located within the chosen ROI, a series of filters was applied to the acquired image stack in the X-Y (space) and Z (time) dimensions. First, for each pixel, a moving average filter with a 3-point span was used to smooth the temporal profile of the SF-iGluSnFR responses. Next, a bandpass Gaussian filter $(0.5 \mathrm{~Hz}-30$ $\mathrm{Hz}$ ) was applied in order to amplify the SF-iGluSnFR signal, revealing glutamate release sites by removing background fluorescence (Fig. 1C, D and Movie S1). Next, a median filter (3x3 pixels) was used to reduce the spatial high-frequency noise component and to improve the robustness of automatic detection of active boutons. Finally, a maximal projection of the 
filtered stack was obtained in order to visualise all regions where glutamate release events were detected, regardless of the rate of their occurrence. Positions of putative glutamate release sites on the maximal projection image were automatically detected using Find Maxima ImageJ plugin and a set of circular ROIs centred on the detected maxima (diameter 5 pixels, $\sim 0.9 \mu \mathrm{m}$ ) was created. The detection threshold was chosen in a such way that all putative release sites were included, along with false-positive sites originating from background noise (these were excluded during quantal analysis stage as described below).

\section{Detection of quantal glutamate release events}

To determine the timings and the amplitudes of vesicular release events the SF-iGluSnFR fluorescence signal from each selected ROI was first filtered using a $0.5 \mathrm{~Hz}-30 \mathrm{~Hz}$ bandpass Gaussian filter, which allowed us to remove the baseline drift and the high frequency noise. This was followed by deconvolution of the experimentally determined average unitary SFiGluSnFR response, approximated as an instantaneous rise followed by an exponential decay $f_{i \text { GluSnFR }}^{\text {unitary }}(t)=e^{-t / \tau} \quad$ with a decay time $\tau=68$ ms (Fig. $\mathrm{S} 1$ ): $f_{\text {deconv }}(t)=F^{-1}\left[F\left(f_{i G l u S n F R}^{\text {filtered }}(t)\right) / F\left(f_{\text {iGluSnFR }}^{\text {unitary }}(t)\right)\right]$, where $F$ is the discrete Fourier transform and $F^{-1}$ is the inverse Fourier transform functions (MATLAB). The obtained deconvolved trace was further filtered using a $0.5 \mathrm{~Hz}-30 \mathrm{~Hz}$ bandpass Gaussian filter to improve signalto-noise ratio. In line with previous reports ${ }^{17,29}$, the all-point histogram of the deconvolved trace could be well-approximated by a single Gaussian centred at zero: $\frac{A}{\sigma_{B N} \sqrt{2 \pi}} \exp \left(\frac{-x^{2}}{2{\sigma_{B N}}^{2}}\right)$ (Fig. S2A). The obtained standard deviation $\sigma_{B N}$ (characterising the level of baseline noise) was then used to set the bouton-specific threshold for the detection of quantal events at $\theta=4 \sigma_{B N}{ }^{29}$. The amplitudes and the timings of individual release events were then determined at local maxima on the filtered deconvolved trace above the threshold.

\section{Quantal analysis}

To determine the amplitude of deconvolved SF-iGluSnFR signal corresponding to release of a single quanta $(q)$ and the distribution of quantal vesicular release events in each bouton, we generated a quasi-continuous amplitude histogram using a bootstrapping procedure with added bouton specific noise ( $m=100,000$ simulations). The simulated values were obtained by randomly selecting an experimentally determined amplitude and adding a random number form a Gaussian distribution with a standard deviation $\sigma_{B N}$. The positions of quantal peaks on the obtained histogram were fitted using a finite mixture model consisting of the sum of 4 Gaussians: $\sum_{i=1}^{4} \frac{A_{i}}{\sigma \sqrt{2 \pi}} \exp \left(\frac{-\left(x-\mu_{i}\right)^{2}}{2 \sigma^{2}}\right)$, where $A_{i}$ is the amplitude of the $i$ th peak, $\mu_{i}=\sum_{k=1}^{i} \lambda^{(k-1)} \mu_{1}$ is the average SF-iGluSnFR event amplitude corresponding to simultaneous release of $i$ vesicles, $\lambda$ is a factor that accounts for possible progressive saturation of SFiGluSnFR signals during multi-vesicular release events, $\sigma^{2}=\sigma_{B N}{ }^{2}+\sigma_{A N}{ }^{2}$ is the variance of 
each peak, and $\sigma_{A N}{ }^{2}$ is the noise component associated with variability of SF-iGluSnFR amplitudes (including the variability caused by a random jitter between the timings of release events and the camera exposure cycle, Fig. S3). The use of bootstrapping allowed us to eliminate the error associated with the sensitivity of fitting procedure to the histogram bin size. The mean value of the saturation factor $\lambda$ estimated across $n=1,130$ boutons from 16 WT neurons was 0.9 (coefficient of variation 0.15 ). Thus, in line with a previous study in ribbon synapses ${ }^{17}$, the amplitudes of deconvolved SF-iGluSnFR signals provided a nearly linear readout for multi-vesicular release.

\section{Temporal resolution and sensitivity of quantal analysis}

To estimate the temporal resolution and the sensitivity of SF-iGluSnFR quantal analysis we simulated a set of SF-iGluSnFR synaptic responses $(n=2,000)$ using the experimentally determined signal-to-noise ratio (Fig. S2B) and processed the obtained traces using the filtering and deconvolution procedures described above. Analysis of simulated traces verified that temporal resolution of detection of vesicular release events was primarily limited by the camera acquisition rate ( $4 \mathrm{~ms}$ ). The simulations confirmed that the use of $\theta=4 \sigma_{B N}$ detection threshold effectively abolished the presence of false-positive events ( 5 false positive in 1000 simulated traces). However, the fraction of false-negative (missed) events was increased with the decrease of signal-to-noise ratio (Fig. S3E). We therefore excluded boutons with signal-tonoise ratio below $5 \sigma_{B N}(\sim 17 \%$ of all boutons, which are likely to be boutons outside the focal plane), thus limiting the fraction of false-negative events to $20 \%$.

\section{Sub-pixel localisation of vesicular release sites}

For each active bouton a $21 \times 21$-pixel ROI $(3.85 \times 3.85 \mu \mathrm{m})$, centred at the corresponding intensity maximum on the maximal projection filtered stack (Fig. 1D), was extracted from the raw SF-iGluSnFR image stack. If the distance between two neighbouring boutons was less than 16 pixels $(\sim 3 \mu \mathrm{m})$ they were excluded from the analysis.

To increase the signal-to-noise ratio, the extracted image stacks were pre-processed pixel-bypixel in time domain. The traces were processed using a bandpass Gaussian filter $(0.5 \mathrm{~Hz}-30$ $\mathrm{Hz}$ ), which was followed by deconvolution of the unitary SF-iGluSnFR response from the filtered signal, as described in the 'Identification of active boutons' section. Vesicular release event was considered to contain a single quantum if its amplitude was below a $1.25 q$ threshold (determined using quantal analysis). For each single quantal release, an 'event image' was calculated by averaging 3 frames from the deconvolved image stack centred at the response peak (Fig. 3B). The event image was then used for sub-pixel localisation of the vesicular release site with the ThunderSTORM ImageJ plugin ${ }^{30}$.

The analysis consisted of two steps: finding the approximate position of the release site and its subsequent sub-pixel localisation. The event image was filtered using a wavelet filter, with a B-spline function of order 3 and a scale of 2 . The approximate position of the release site was determined by finding a local maximum on the filtered image (intensity is greater than the specified threshold and at the same time greater than or equal to the intensities of 8 neighbouring pixels). Because the signal-to-noise ratio varied among synapses, the optimal threshold value was set independently for each bouton. For this purpose, we generated a 
'background noise' image stack consisted of 1,000 images. Each 'background noise' image was obtained by averaging 3 randomly selected frames from the deconvolved image stack, which were separated from the nearest release event by at least 15 frames (or $60 \mathrm{~ms}$ ). The optimal bouton-specific threshold was then determined by running the analysis iteratively on the 'background noise' image stack, starting from a high level of intensity and decreasing it by half at every iteration until some events were detected. A second iterative process increased the threshold value in 250 intensity level steps, until no maxima were detected. The determined threshold value was next used to analyse the event images from the same bouton. Event images where the approximate position of the release site could not be determined with the selected threshold were excluded from the analysis. Because we preselected images corresponding to release of single vesicles, we a priori knew that there should be only one spatial maximum in each image, therefore we excluded images if more than one local maximum was detected. Finally, the sub-pixel localisation of single quanta release events that passed all the above selection criteria was performed by fitting an integrated form of 2D Gaussian function into the $7 \times 7$ pixel array centred around the established approximate positions ${ }^{30}$.

\section{Estimation of release sites localisation precision}

The precision of localisation of vesicular release sites depends on several key factors. First as in the case of single molecule localisation microscopy, the localisation accuracy depends on the spatial resolution (point spread function, PSF) of the microscope and on the strength of SFiGluSnFR signal (i.e. the signal-to-noise ratio determined by the number of photons collected for a given release event). In addition to this, the localisation accuracy of release events also depends on the spatial profile of the activated SF-iGluSnFR molecules within a given active zone and on the relative position and tilt of the imaged active zone with respect to the microscope focal plane ${ }^{21}$.

To account for the joint effect of all the above factors we estimated the localisation accuracy using an empirical approach. For each event we generated an 'added noise' image stack (in total 50 images). Each of the images in the 'added noise' stack was a sum of the original event image and a randomly selected image from the 'background noise' image stack described in the 'Sub-pixel localisation of vesicular release sites' above. We next applied the localisation analysis to individual images from the 'added noise' stack and calculated the average position of all fits (Fig. S6B). The distance $\delta$ between the initial fit and the average position obtained from the 'added noise' image stack was used as an empirical estimate of the localisation accuracy (Fig. S6 C, D) and events with $\delta$ above the $100 \mathrm{~nm}$ threshold were excluded from analysis.

To test if $\delta$ represents a realistic estimate of the localisation accuracy we performed similar analysis using artificial computer-simulated images. The rational was that in this case we $a$ priory knew the true locations of release sites. The simulated 'event images' were obtained using the following steps. We first simulated a spatio-temporal SF-iGluSnFR profiles corresponding to single vesicle fusion events using a general function form:

$$
F(x, y, t)=F_{B G}(x, y)+\Delta F \exp \left(\frac{-\left(x-x_{0}\right)^{2}-\left(y-y_{0}\right)^{2}}{2 \sigma_{x y}}\right) \exp \left(\frac{-\left(t-t_{0}\right)^{2}}{\tau}\right),
$$

where $\left(x_{0}, y_{0}\right)$ and $t_{0}$ are coordinates and timing of a release event; $\tau=68 \mathrm{~ms}$, SF-iGluSnFR 
decay rate (Fig. S1), $\sigma_{x y}$, spatial width of SF-iGluSnFR response (randomly selected from the experimentally determined range $250-450 \mathrm{~nm}), F_{B G}(x, y)$ and $\Delta F$ background SF-iGluSnFR fluorescence and the amplitude of responses respectively (randomly selected from the recoded boutons). The obtained $F(x, y, t)$ responses were mapped on the experimental spatio-temporal grid (183.3 $\mathrm{nm}$ in space and $4 \mathrm{~ms}$ in time domain) and noise was added using a randomly selected value of signal-to-noise ratio from the experimental data set (Fig. S2B). Finally, locations of simulated vesicular release events were fitted using the same routine as for the experimental data. The analysis of artificial images verified that $\delta$ provides an accurate estimate of localisation precision ( $50-100 \mathrm{~nm}$ range). Furthermore, the computer simulations demonstrated that the use of the averaged position determined from the 'added noise' stack provides a more reliable estimate for the location of release site. Therefore, we used this improved averaged fit in the analysis of the relative distributions of synchronous and asynchronous release events in Fig. 3F-G.

\section{Hierarchical cluster analysis}

Hierarchical cluster analysis was used to investigate the distribution of synchronous and asynchronous events among different release sites (Fig. 3F and Fig. S7). The built-in MATLAB functions 'linkage' and 'cluster' were used to best group events based on a distance threshold, allowing individual release cites to be defined by events located within $100 \mathrm{~nm}$ of the cluster centroid (in accordance with the precision of localisation of individual exocytosis events). The same algorithm was applied to identify synapses with two active zones (see examples in Fig S7). If boutons displayed event clusters with centroids distanced by $700 \mathrm{~nm}$ or more, they were defined as having two active zones and were excluded from spatial analysis.

\section{Computational modelling of synchronous and asynchronous release within the active zone}

Based on the available electron microscopy data ${ }^{3,4,12,31,32}$, we considered a typical active zone of an area $S=0.12 \mu \mathrm{m}^{2}$, that contained two VGCCs clusters (located at the active zone periphery) and $N_{r e l}=12$ of vesicular release cites that were randomly distributed across the active zone (Fig. 4C,D). The modelling of three-dimensional action potential-evoked presynaptic $\mathrm{Ca}^{2+}$ dynamics and of $\mathrm{Ca}^{2+}$ activation of vesicular release was performed using the presynaptic terminal model implemented in the Virtual Cell (VCell) simulation environment (http://vcell.org) and custom-developed MATLAB (MathWorks) scripts as described in detail in our previous work ${ }^{33-35}$. Briefly, we simulated action potential-evoked spatio-temporal $\mathrm{Ca}^{2+}$ dynamics in the vicinity of a VGCC cluster (containing 7 P/Q-type, $8 \mathrm{~N}$-type, and 1 R-type VGCCs) in the presence of the three endogenous buffers (calmodulin, Calbindin D28k and ATP). We next computed the probability maps for synchronous release in response to $\mathrm{Ca}^{2+}$ influx at each of the two VGCC clusters $\left(p 1_{v}(x, y)\right.$ and $\left.p 2_{v}(x, y)\right)$ using the six-state allosteric model of $\mathrm{Ca}^{2+}$ activation of vesicle fusion ${ }^{36}$. As there was virtually no spatial overlap between $p 1_{S}(x, y)$ and $p 2_{S}(x, y)$ we approximated the synchronous release probability map as $p_{S}(x, y)=p 1_{S}(x, y)+p 2_{S}(x, y)$. Synchronous release was then simulated by considering the overall release probability as the product $p_{S}(x, y) \cdot p_{o c}$, where $p_{o c}$ is the probability that a 
given release site is occupied by a RRP vesicle. Because vesicular release during $5 \mathrm{~Hz}$ stimulation was on average depressed by $\sim 2$ fold in comparison to the response at the $1^{\text {st }}$ action potential, we considered $p_{o c}=0.5$. Probability of asynchronous release was assumed to be independent on the release site location and was estimated as $p_{A}=n_{A} / N_{\text {rel }}=0.01$, where $n_{A}$ is the average rate of asynchronous release per action potential during $5 \mathrm{~Hz}$ stimulation $\left(n_{A} \sim 0.12\right.$, Fig. $\left.1 \mathrm{G}\right)$.

\section{Data Inclusion and Exclusion Criteria}

In addition to the exclusion criteria described in the above sections we applied the following selection criteria. In Fig. 2A, B, boutons with at least 2 release events were selected to allow meaningful estimation of $n_{A} / n_{T}$. Similarly, in Fig. 2C boutons with at least 2 synchronous events were selected to allow estimation of $P P R$. Previous work ${ }^{21}$, where vGlut1-pHluorin probe was used for sub-pixel localisation of vesicular release sites, demonstrated that positions of release events can be reliably fitted only within $\sim 100 \mathrm{~nm}$ from the imaging plane in $\mathrm{z}$ direction and that selection of boutons with at least 5 detected events effectively limits the analysis to a sub-population of active zones that are mostly parallel to the image plane (within a $20^{\circ}$ tilt). We therefore applied the same selection criteria in our analysis in Fig.3. Furthermore, in order to compare the relative locations of synchronous and asynchronous release events (Fig. $3 \mathrm{~F}-\mathrm{G})$ only boutons that contained both event types were included.

\section{Statistical analysis}

The distribution of data in each set of experiments was first tested for normality using the Shapiro-Wilk test. The similarity of variances between each group of data was tested using the F test. Normally distributed data were presented as mean \pm s.e.m., each plot also contained the individual data points. Student's $t$ tests for group means or paired t tests were used as indicated. The data sets that failed the normality test were presented using box-and-whisker plots (box $25^{\text {th }}-75^{\text {th }}$ percentiles, whiskers $10^{\text {th }}-90^{\text {th }}$ percentiles), each plot also contained the individual data points and compared using Mann-Whitney $U$ test. The detailed statistical analysis is presented in Supplementary Table 1. No statistical methods were used to pre-determine sample sizes, but our sample sizes were similar to those reported in previous publications that use similar techniques ${ }^{16,17,34}$. Data analysis was performed blind to the conditions and genotype tested. All statistical tests were performed using SigmaPlot 11 (Systat Software) and MATLAB (MathWorks) software packages.

\section{Acknowledgments}

We are grateful to Dimitri Kullmann, James Rothman, Shyam Krishnakumar and Christopher Kushmerick for reading the manuscript and providing critical feedback.

\section{Author contributions}

Conceptualization: PRFM, KEV

Performing experiments: PRFM, ET, HL, DK

Development of data analysis framework: PRFM, CGZC, YT, KEV

Computational modelling: YT, KEV

Writing - original draft: PRFM, YT, KEV 
Writing - review and editing: PRFM, ET, HL, DK, CGZC, YT, KEV

Funding acquisition: YT, KEV

Project supervision: KEV

\section{Competing interests}

The authors declare no competing interests.

\section{Data and materials availability}

All the data are available upon request from the corresponding authors.

\section{Funding}

The Wellcome Trust Strategic Award 104033/z/14/z (KEV)

Epilepsy Research UK Project Grant P1806 (KEV)

The Wellcome Trust PhD Studentship 203795/Z/16/Z (HL)

Medical Research Council UK Project Grant MR/T002786/1 (YT and KEV) 


\section{References}

1. Kaeser,P.S. \& Regehr,W.G. Molecular mechanisms for synchronous, asynchronous, and spontaneous neurotransmitter release. Annu. Rev. Physiol. 76:333-63. doi: 10.1146/annurev-physiol-021113-170338. Epub@2013 Nov 21., 333-363 (2014).

2. Tang,A.H. et al. A trans-synaptic nanocolumn aligns neurotransmitter release to receptors. Nature. 536, 210-214 (2016).

3. Brockmann,M.M. et al. Functional architecture of the synaptic transducers at a central glutamatergic synapse. BioRxiv2020 (2020).

4. Li,S. et al. Asynchronous release sites align with NMDA receptors in mouse hippocampal synapses. Nat. Commun. 12, 677 (2021).

5. Goda,Y. \& Stevens,C.F. Two components of transmitter release at a central synapse. Proc. Natl. Acad. Sci. U. S. A. \%20;91, 12942-12946 (1994).

6. Huson,V. \& Regehr,W.G. Diverse roles of Synaptotagmin-7 in regulating vesicle fusion. Curr. Opin. Neurobiol. 63:42-52. doi: 10.1016/j.conb.2020.02.006., 42-52 (2020).

7. Deng,S. et al. Regulation of Recurrent Inhibition by Asynchronous Glutamate Release in Neocortex. Neuron. 105, 522-533 (2020).

8. Li,J., Deng,S., He,Q., Ke,W., \& Shu,Y. Asynchronous Glutamate Release at Autapses Regulates Spike Reliability and Precision in Mouse Neocortical Pyramidal Cells. Cereb. Cortex.bhaa361 (2020).

9. Luo,F. \& Sudhof,T.C. Synaptotagmin-7-Mediated Asynchronous Release Boosts High-Fidelity Synchronous Transmission at a Central Synapse. Neuron. 94, 826-839 (2017).

10. Hefft,S. \& Jonas,P. Asynchronous GABA release generates long-lasting inhibition at a hippocampal interneuron-principal neuron synapse. Nat. Neurosci. 8, 1319-1328 (2005).

11. Turecek,J. \& Regehr,W.G. Neuronal Regulation of Fast Synaptotagmin Isoforms Controls the Relative Contributions of Synchronous and Asynchronous Release. Neuron. 101, 938-949 (2019).

12. Kusick,G.F. et al. Synaptic vesicles transiently dock to refill release sites. Nat. Neurosci. 23, 1329-1338 (2020).

13. Tagliatti,E. et al. Synaptotagmin 1 oligomers clamp and regulate different modes of neurotransmitter release. Proc. Natl. Acad. Sci. U. S. A. 117, 3819-3827 (2020).

14. Marvin,J.S. et al. Stability, affinity, and chromatic variants of the glutamate sensor iGluSnFR. Nat. Methods. 15, 936-939 (2018).

15. Jensen,T.P. et al. Multiplex imaging relates quantal glutamate release to presynaptic $\mathrm{Ca}(2+)$ homeostasis at multiple synapses in situ. Nat. Commun. 10, 1414-09216 (2019).

16. Durst,C.D. et al. High-speed imaging of glutamate release with genetically encoded sensors. Nat. Protoc. 14, 1401-1424 (2019).

17. James,B., Darnet,L., Moya-Diaz,J., Seibel,S.H., \& Lagnado,L. An amplitude code transmits information at a visual synapse. Nat. Neurosci. 22, 1140-1147 (2019). 
18. Geppert,M. et al. Synaptotagmin I: a major Ca2+ sensor for transmitter release at a central synapse. Cell. 79, 717-727 (1994).

19. Sabater,V.G., Rigby,M., \& Burrone,J. Voltage-gated potassium channels ensure action potential shape fidelity in distal axons. BioRxiv2020 (2020).

20. Ermolyuk,Y.S. et al. Independent Regulation of Basal Neurotransmitter Release Efficacy by Variable $\mathrm{Ca}<\sup >2+</$ sup $>$ Influx and Bouton Size at Small Central Synapses. PLoS Biol 10, e1001396 (2012).

21. Maschi,D. \& Klyachko,V.A. Spatiotemporal Regulation of Synaptic Vesicle Fusion Sites in Central Synapses. Neuron. 94, 65-73 (2017).

22. Rozov,A., Burnashev,N., Sakmann,B., \& Neher,E. Transmitter release modulation by intracellular $\mathrm{Ca} 2+$ buffers in facilitating and depressing nerve terminals of pyramidal cells in layer $2 / 3$ of the rat neocortex indicates a target cell-specific difference in presynaptic calcium dynamics. J. Physiol. 531, 807-826 (2001).

23. Grauel,M.K. et al. RIM-binding protein 2 regulates release probability by finetuning calcium channel localization at murine hippocampal synapses. Proc. Natl. Acad. Sci. U. S. A 113, 11615-11620 (2016).

24. Kaeser,P.S. et al. RIM Proteins Tether $\mathrm{Ca}(2+)$ Channels to Presynaptic Active Zones via a Direct PDZ-Domain Interaction. Cell 144, 282-295 (2011).

25. Markram,H., Wang,Y., \& Tsodyks,M. Differential signaling via the same axon of neocortical pyramidal neurons. Proc. Natl. Acad. Sci. U. S. A. 95, 5323-5328 (1998).

26. Reyes,A. et al. Target-cell-specific facilitation and depression in neocortical circuits. Nat. Neurosci. 1, 279-285 (1998).

27. Edelstein,A.D. et al. Advanced methods of microscope control using muManager software. J. Biol. Methods. 1, 10 (2014).

28. Schneider,C.A., Rasband,W.S., \& Eliceiri,K.W. NIH Image to ImageJ: 25 years of image analysis. Nat. Methods. 9, 671-675 (2012).

29. Pernia-Andrade,A.J. et al. A deconvolution-based method with high sensitivity and temporal resolution for detection of spontaneous synaptic currents in vitro and in vivo. Biophys. J. 103, 1429-1439 (2012).

30. Ovesny,M., Krizek,P., Borkovec,J., Svindrych,Z., \& Hagen,G.M. ThunderSTORM: a comprehensive ImageJ plug-in for PALM and STORM data analysis and super-resolution imaging. Bioinformatics. 30, 2389-2390 (2014).

31. Rebola,N. et al. Distinct Nanoscale Calcium Channel and Synaptic Vesicle Topographies Contribute to the Diversity of Synaptic Function. Neuron. \%20;104, 693-710 (2019).

32. Holderith,N. et al. Release probability of hippocampal glutamatergic terminals scales with the size of the active zone. Nat. Neurosci. 15, 988-997 (2012).

33. Timofeeva,Y. \& Volynski,K.E. Calmodulin as a major calcium buffer shaping vesicular release and short-term synaptic plasticity: facilitation through buffer dislocation. Front Cell Neurosci. 9:239. doi: 10.3389/fncel.2015.00239. eCollection@2015., 239 (2015).

34. Ermolyuk,Y.S. et al. Differential triggering of spontaneous glutamate release by P/Q-, N- and R-type Ca(2+) channels. Nat. Neurosci. 16, 1754-1763 (2013). 
35. Chamberland,S., Timofeeva,Y., Evstratova,A., Volynski,K., \& Toth,K. Action potential counting at giant mossy fiber terminals gates information transfer in the hippocampus. Proc. Natl. Acad. Sci. U. S. A. 115, $7434-7439$ (2018).

36. Lou,X., Scheuss,V., \& Schneggenburger,R. Allosteric modulation of the presynaptic $\mathrm{Ca} 2+$ sensor for vesicle fusion. Nature. 435, 497-501 (2005). 


\section{Figures}

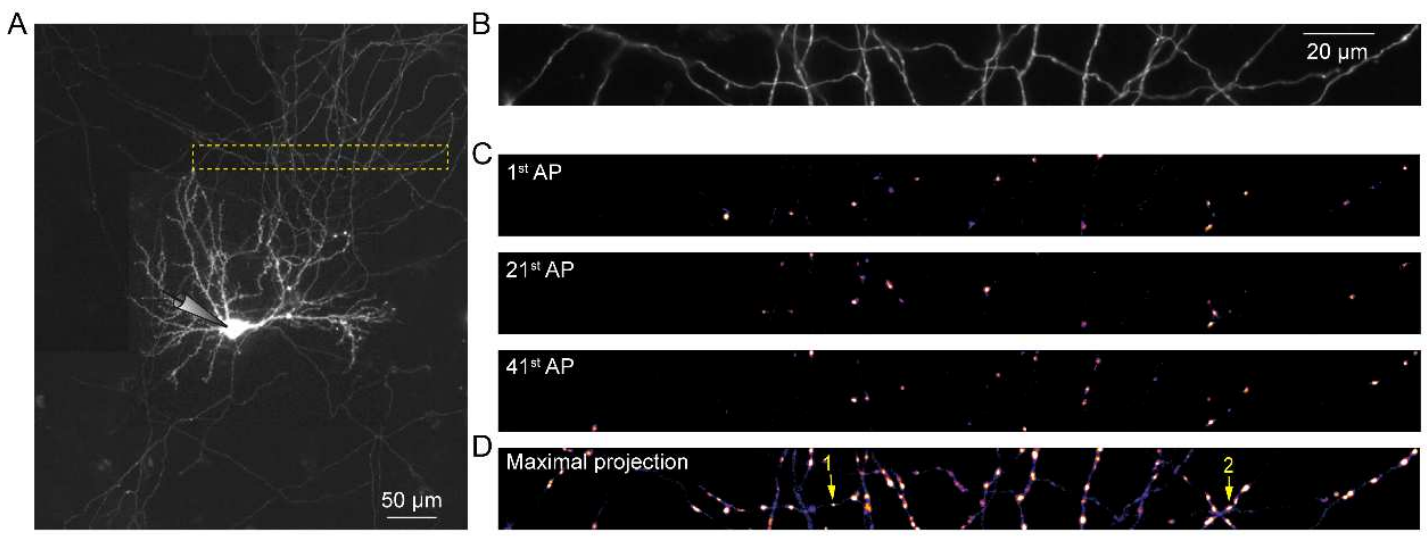

E APs
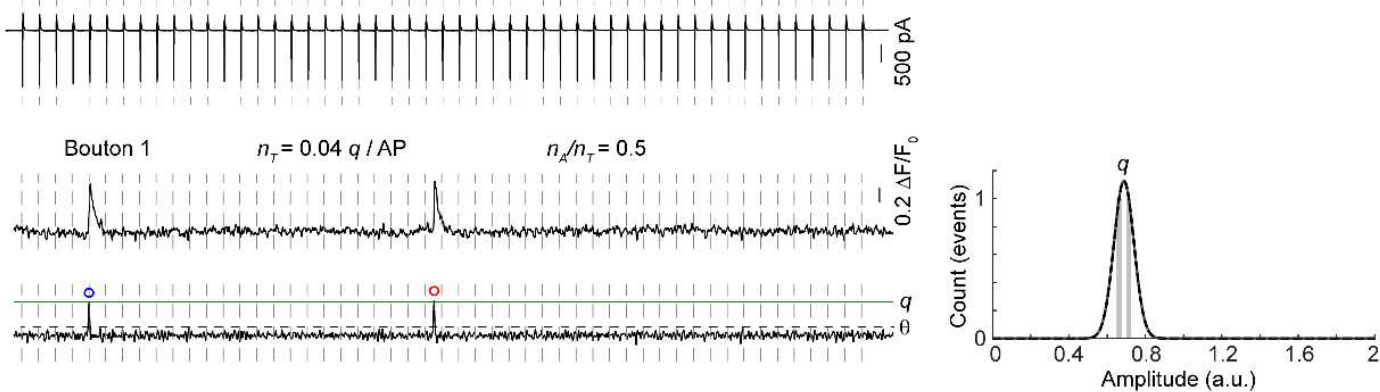

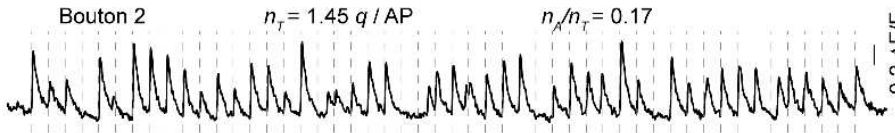
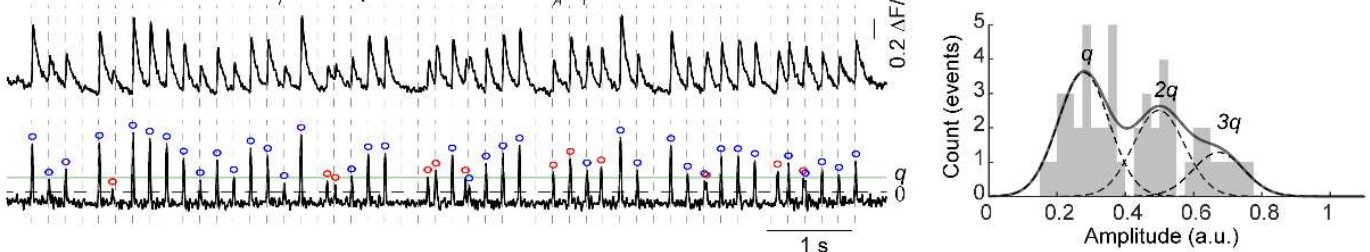

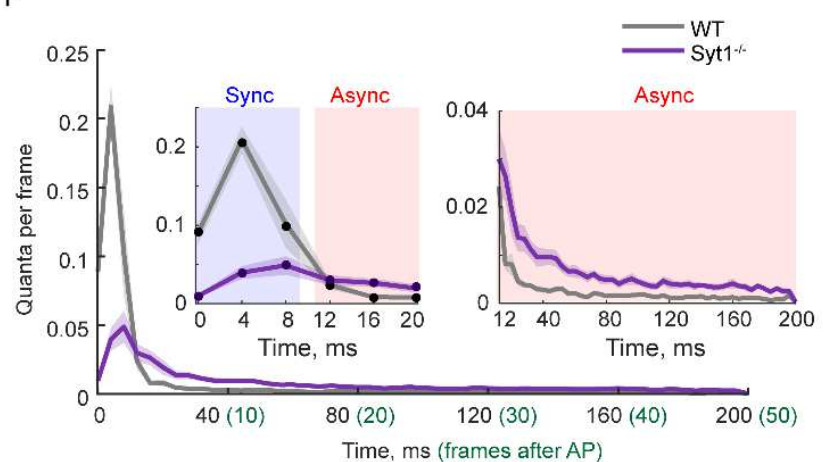

G
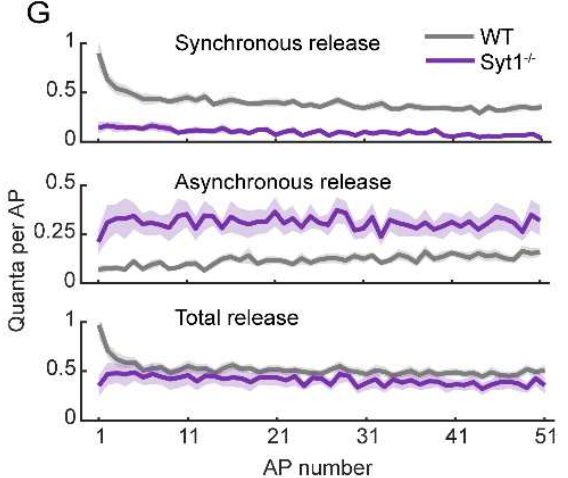

Fig. 1. SF-iGluSnFR fluorescence imaging of quantal synchronous and asynchronous release in tens of individual presynaptic boutons supplied by a single axon.

(A to D) A detailed illustration of a typical experiment. (A) Reconstructed mosaic image of a pyramidal neuron expressing the SF-iGluSnFR probe. (B) A region of interest (ROI) in the axonal arbour selected for imaging of presynaptic SF-iGluSnFR responses corresponding to the yellow box in (A). (C) Heat maps of SF-iGluSnFR responses to the $1^{\text {st }}, 21^{\text {st }}$, and $41^{\text {st }}$ action potentials (APs) during $5 \mathrm{~Hz}$ train of 51 APs. The images are averages of 3 frames from a band- 
pass filtered image stack immediately after each action potential (see Methods and Movie S1). (D) Maximal projection of the band-pass filtered image stack revealing locations of all presynaptic boutons that released at least one vesicle during the stimulation train.

(E) Analysis of quantal SF-iGluSnFR responses in two representative boutons (Boutons 1 and 2 in D). Left, somatic action potential escape currents time-aligned with band-pass filtered and deconvolved SF-iGluSnFR signals. Quantal release events were identified as local maxima on the deconvolved traces located above the threshold $\theta$ (horizontal dashed lines) corresponding to 4 standard deviations of the background noise (see Figs. S2, S3 and Methods). Right, quantal analysis. To determine the amplitude of SF-iGluSnFR signal corresponding to release of a single vesicle ( $q$, green lines on deconvolved traces) the positions of peaks on the amplitude histograms were fitted with a sum of 4 Gaussian functions (black lines). This was then used to calculate in each bouton the total number of vesicles released per action potential (release rate $\left.n_{T}\right)$ and the fraction of asynchronous release events $\left(n_{A} / n_{T}\right)$ (see below and also Figs. S4, S5 for more examples).

(F) Comparison of evoked vesicular release kinetics in wild type (WT) and Syt1 $1^{-/-}$neurons. The traces were obtained by first averaging in individual boutons the number of quanta detected in individual frames following each of 51 action potentials in the train (frames 0 to 50,4 $\mathrm{ms} /$ frame). This was followed by calculating the cell-averaged responses (range $14-162$ boutons per cell) and finally the mean responses across all recoded neurons. Based on the distinct biphasic time-course of vesicular release in wild type neurons, the time threshold between synchronous and asynchronous release events was set at $\sim 10 \mathrm{~ms}$ after the somatic action potential (i.e. at the border between $2^{\text {nd }}$ and $3^{\text {rd }}$ frames, see main text for details). Using this criterion, we then classified individual release events either as synchronous (blue circles on the deconvolved traces) or asynchronous (red circles).

(G) Comparison of synchronous, asynchronous and total release in wild type and Syt1 $1^{-/}$ neurons during the $5 \mathrm{~Hz}$ stimulation train. Traces represent the average number of vesicular quanta released at each action potential in a single presynaptic bouton (mean $\pm \mathrm{SEM}$ ). Wild type, $\mathrm{N}=16$ cells; $\mathrm{Syt}^{-/-}, \mathrm{N}=11$ cells. 

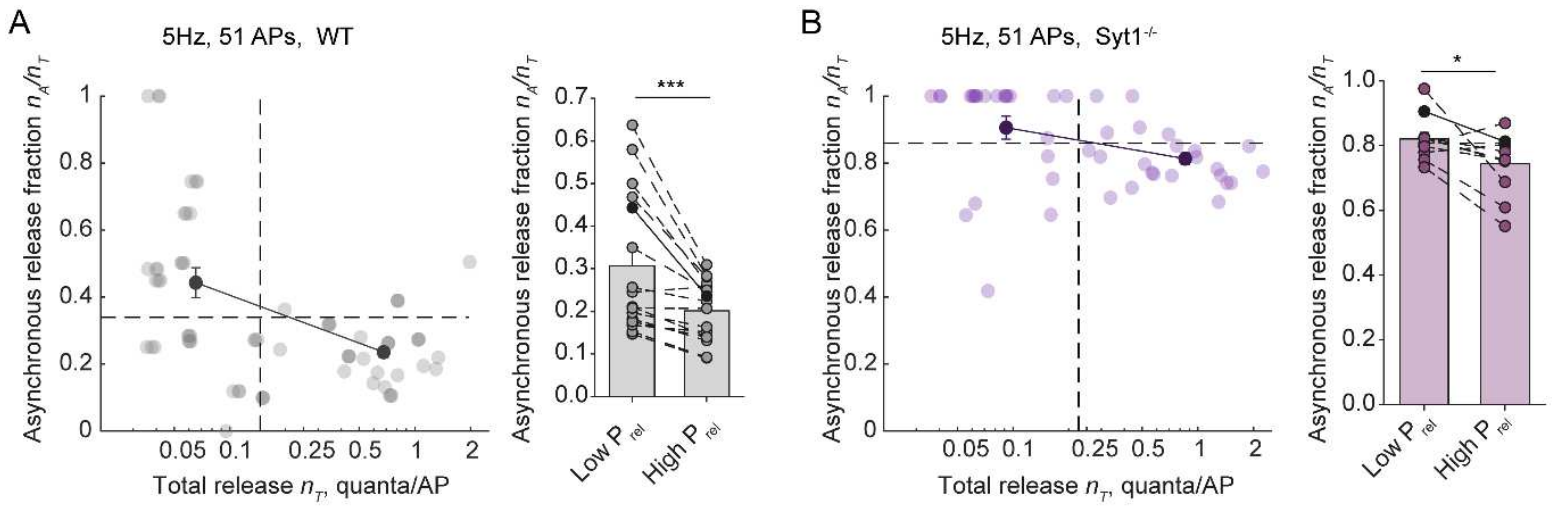

C

Paired-pulse protocol: $10 \times 2 \mathrm{APs}$ at $2 \mathrm{OHz}$, WT

(i)

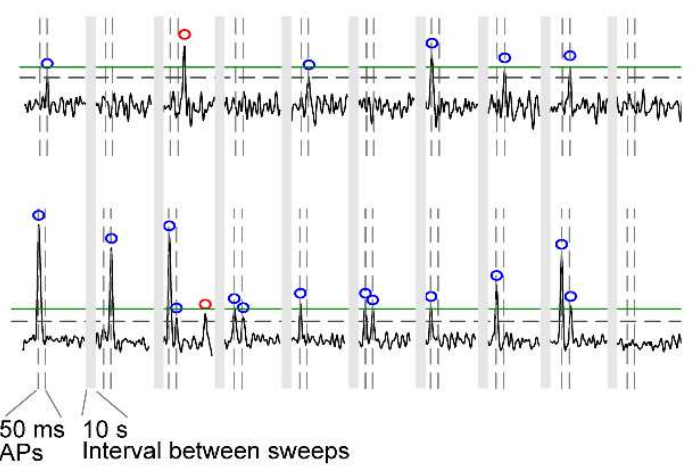

(ii)
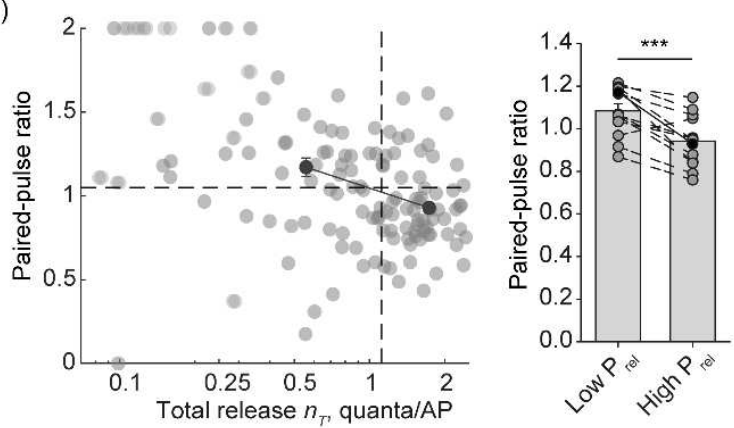

(iii)

(iv)
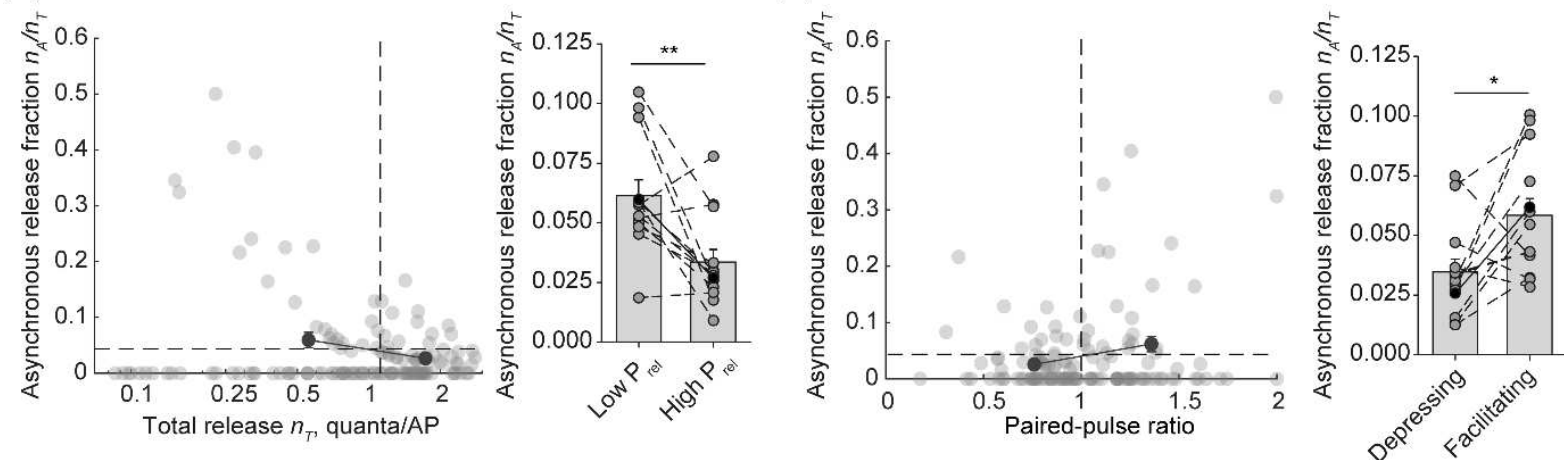

Fig. 2 Asynchronous release is elevated in parallel with short-term facilitation in low release probability synapses.

(A, B) Analysis of synchronous and asynchronous release heterogeneity among presynaptic outputs of individual wild type (A) and $\mathrm{Syt1}^{-/-}$(B) neurons during $5 \mathrm{~Hz}$ train of 51 action potentials. Left panels, the relationship between asynchronous release fraction $n_{A} / n_{T}$ and the overall vesicular release rate $n_{T}$ among boutons in a representative wild type (WT, $\mathrm{n}=68$ boutons) and a Syt1 ${ }^{-/}(\mathrm{n}=46$ boutons $)$ neurons. Horizontal dashed lines depict the average fractions of asynchronous release in each cell. Vertical dashed lines mark the median values for $n_{T}$, which were used as thresholds to split boutons into groups with low and high $P_{r e l}$. Black dots with error bars show the average values of $n_{A} / n_{T}$ in low and high $P_{\text {rel }}$ groups. Right, bar and dot summary plots showing increase of asynchronous release fraction in low $P_{r e l}$ synapses 
across recorded cells ( $\mathrm{N}=16$ wild type and $\mathrm{N}=11 \mathrm{Syt}^{-/-}$neurons). Data from individual cells are connected by dashed lines, black points - data from the example cells.

(C) Analysis of the relationship between short-term plasticity and synchronicity of glutamate release rate among synaptic outputs of single neurons. (i) Paired-pulse stimulation paradigm and synaptic responses recoded in 2 representative boutons. As in Fig.1 horizontal green lines show the amplitude of SF-iGluSnFR signal corresponding to release of a single vesicle quanta (1q). Vertical dashed lines depict action potential timings. Grey boxes, $10 \mathrm{~s}$ inter-sweep interval. Blue and red circles mark synchronous and asynchronous release events respectively. (ii - iv) The relationships between (ii) paired pulse ratio $(P P R)$ and the overall release rated $n_{T}$ ( PPR in each bouton was estimated as $N_{S 2} /\left(N_{S 1}+N_{S 2}\right)$, where $N_{S 1}$ and $N_{S 2}$ are total numbers of synchronously released quanta at first and second action potentials), (iii) asynchronous release fraction $n_{A} / n_{T}$ and $n_{T}$, and (iv) $n_{A} / n_{T}$ and $P P R$ in a representative cell ( $\mathrm{n}=138$ boutons, left panels), and across recorded cells (bar and dots panels, $\mathrm{N}=13$ cells). Horizontal dashed lines depict the average values of $P P R$ (ii) and $n_{A} / n_{T}$ (iii and iv). Vertical dashed lines in (ii) and (iii) depict the median value of $n_{T}$ in the example cell. Vertical line in (iv) depicts the threshold ( $P P R=1)$ between facilitating and depressing synapses.

$* \mathrm{P}<0.05, * * \mathrm{P}<0.01$ and $* * * \mathrm{P}<0.001$ Paired t-test. To allow visual representation of the overlapping data points, the $\mathrm{x}$ coordinate of each point in the example cell plots shown in this figure was randomly shifted within \pm 0.01 range. 
A
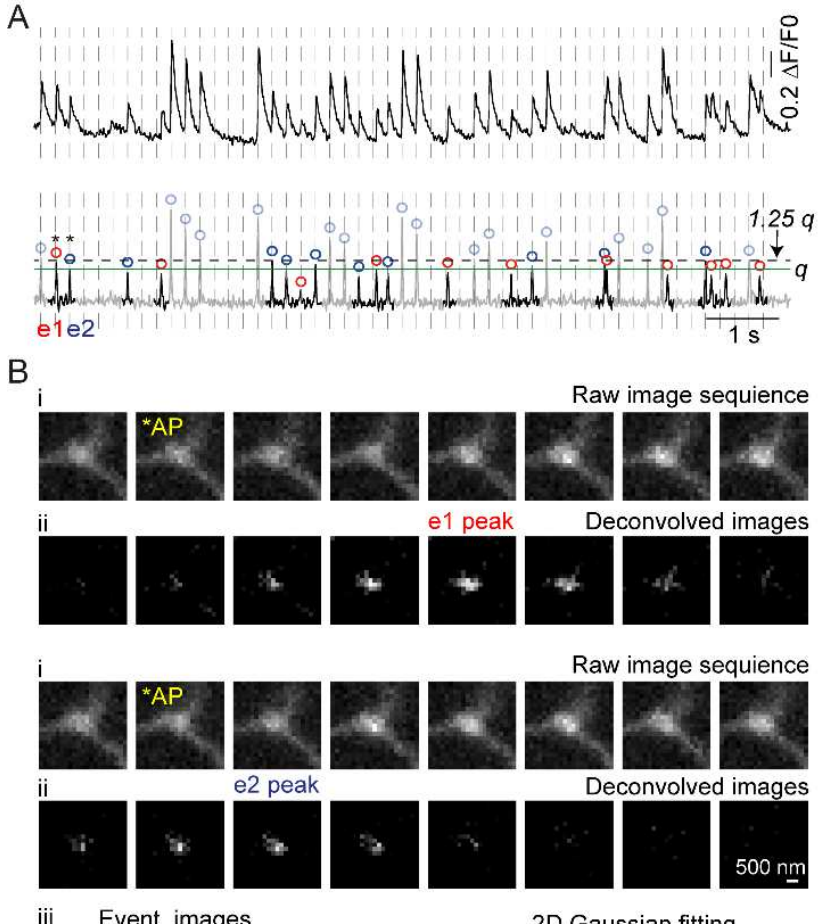

iii Event images
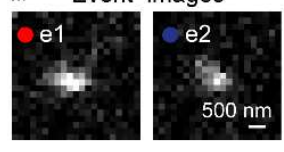

C
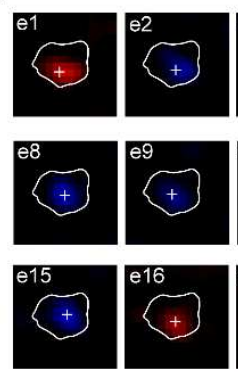

D
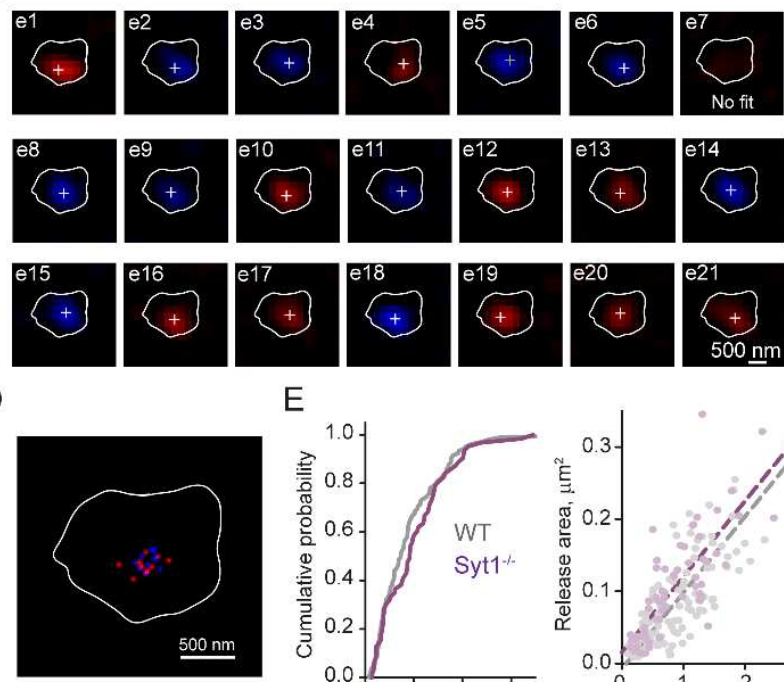

E

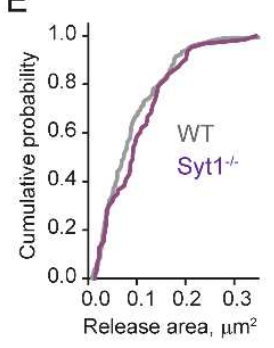

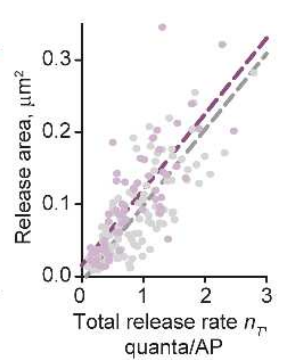

$\mathrm{F}$

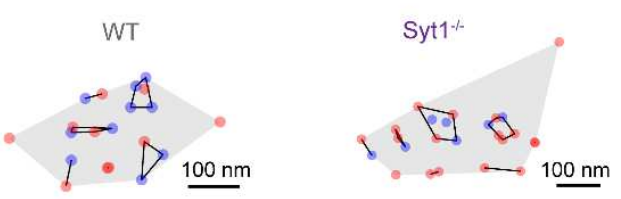

ii

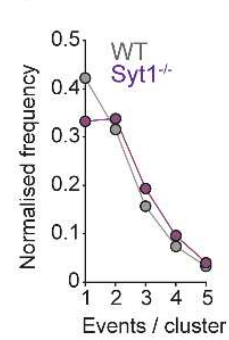

iii iv

。
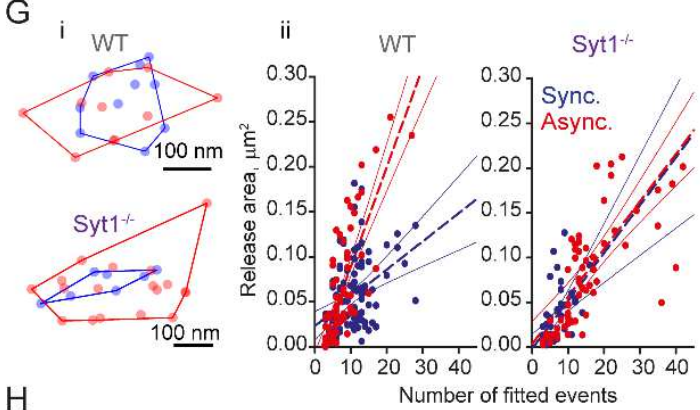

$\mathrm{H}$

Number of fitted events
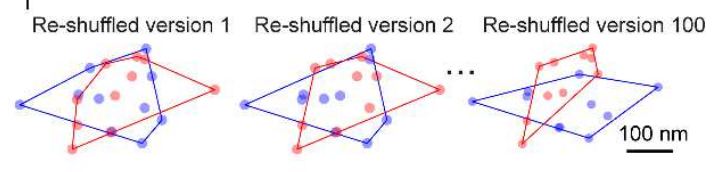

ii

Real Mean Shuffled Ratio Sync. Async.|Sync. Async.|Sync. Async. \begin{tabular}{lll|ll|ll} 
Area & 24.5 & 38.4 & 30.3 & 29.5 & 0.81 & 1.30
\end{tabular}

I
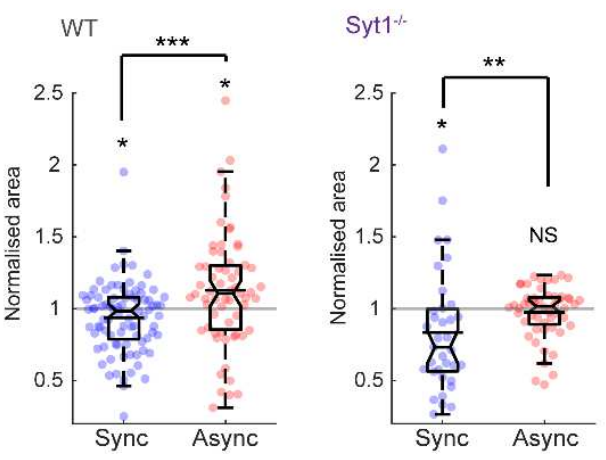

Fig. 3. Spatial distribution of synchronous and asynchronous release events in the active zone.

(A to D) Illustration of sub-pixel localisation analysis for single vesicle exocytosis events.

(A) Raw (top) and deconvolved (bottom) SF-iGluSnFR traces from a representative bouton. Only single vesicle release events (black areas on the deconvolved trace) were included into the localisation analysis. These were selected using 1.25 q amplitude threshold (dashed line), where $q$ (green line) is the quantal size calculated as detailed in Fig. 1E. 
(B) Image pre-possessing in time domain. (i) Raw image sequences before and after event 1 (e1, asynchronous release, 3 frames after the preceding action potential, *AP) and event 2 (e2, synchronous release, 1 frame after the preceding action potential). (ii) Corresponding image sequences after pixel-by-pixel deconvolution of the unitary SF-iGluSnFR response. (iii) 'Event images', obtained by averaging 3 frames centred at the response peak on the deconvolved image stack used for sub-pixel localisation of release locations using 2D Gaussian fitting (Methods).

(C) Sub-pixel localisation (white crosses) of single quantal events selected in (A) (see also Movie S2). Images are after application of a wavelet filter (Methods); e7 could not be localised due to a low signal-to-noise ratio. The bouton outline (white line) was determined by calculating maximal projection of the deconvolved image stack.

(D) Composite image showing relative locations of synchronous (blue) and asynchronous (red) release events within a compact release area.

(E) Distribution of release area sizes and their dependency on the overall release rate $(\mathrm{n}=106$ boutons from $\mathrm{N}=16$ cells for wild type and $\mathrm{n}=64$ boutons from $\mathrm{N}=11$ cells for Syt $1^{-/}$neurons). (F) Hierarchical cluster analysis of vesicular exocytosis sites. (i) Distributions of clustered events (clustering diameter threshold $100 \mathrm{~nm}$, see Methods) in representative wild type (same as in A-D) and Syt1 ${ }^{-/}$boutons. (ii) Distribution of cluster sizes (pooled data from $\mathrm{m}=799$ clusters from $n=106$ boutons in wild type and $m=553$ clusters from $n=64$ boutons in Syt ${ }^{-}$ /- neurons). (iii) Dependency of mixed clusters fraction (containing both synchronous and asynchronous events) on the cluster size. (iv) Increase of synchronous events fraction with cluster size in wild type synapses. Dashed lines, linear regression. Solid lines 95\% confidence intervals.

(G) Comparison of convex hull areas circumventing synchronous or asynchronous events. (i) Representative boutons (as shown in F). (ii) Relationship between the release areas and the number of fitted events in all analysed boutons. Dashed lines, linear regression. Solid lines $95 \%$ confidence intervals.

(H) Illustration of the reshuffling analysis used to compare spatial distributions of synchronous and asynchronous events within individual synapses (representative re-shuffled versions of the wild type bouton shown in A-G, see text for details).

(I) Distribution of the normalised areas for synchronous and asynchronous events in wild type and $\mathrm{Syt}^{-/-}$synapses.

$* \mathrm{p}<0.05, * * * \mathrm{p}<0.001$, NS $\mathrm{p}>0.2$, Mann-Whitney U test. 
A

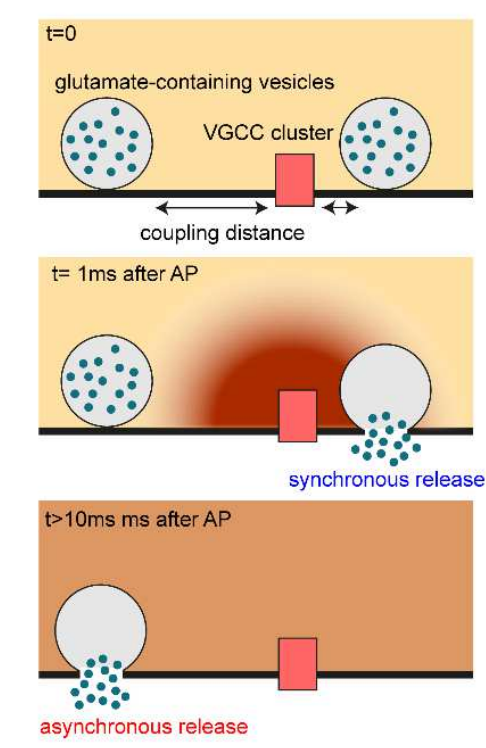

B

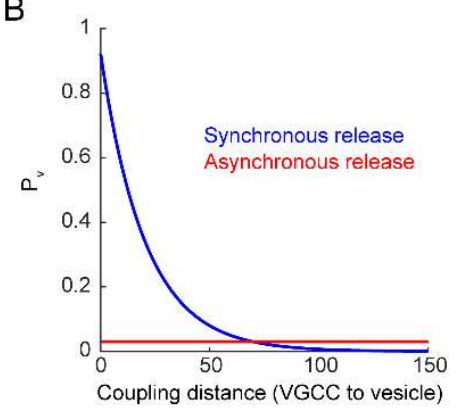

$\mathrm{C}_{\mathrm{i}}$
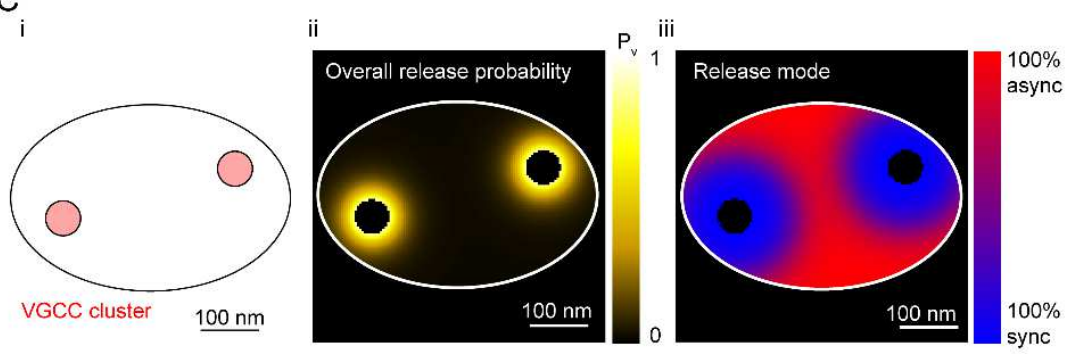

$D_{i}$
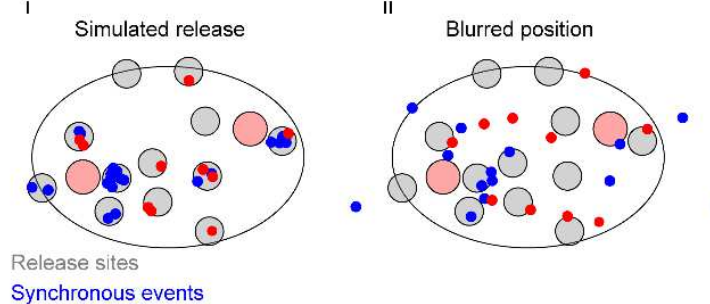

iii

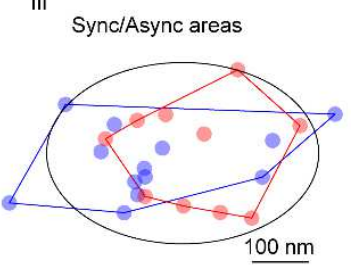

Asynchronous events

E

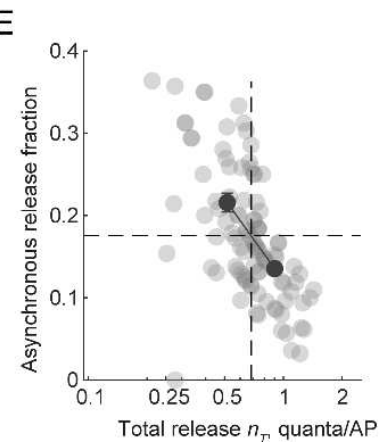

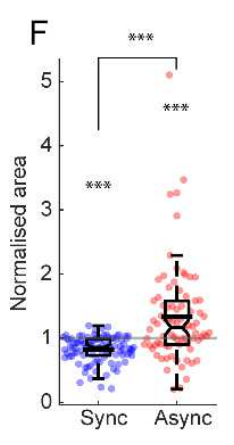

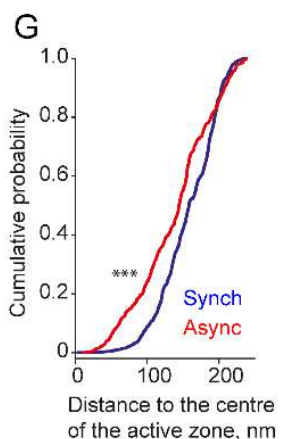

Fig. 4. Modelling of synchronous and asynchronous release implementation of the 'variable coupling distance' model.

(A) $\mathrm{Ca}^{2+}$ triggering of different release modes. Synchronous release occurs within several milliseconds after an action potential and is triggered by transient local $\mathrm{Ca}^{2+}$ nano/microdomains ( $\sim 10-100 \mu \mathrm{M}$ range) in the close vicinity of activated VGCCs $(20-100$ $\mathrm{nm}$ range). In contrast, asynchronous release occurs on a time scale of tens to hundreds of milliseconds and is triggered by longer-lasting global changes in presynaptic $\mathrm{Ca}^{2+}$ concentration $(\sim 1-5 \mu \mathrm{M} \text { range })^{1}$.

(B) Model predicted dependencies of synchronous (blue trace) and asynchronous (red trace) release probabilities on the distance to a VGCC cluster (see Methods).

(C) (i) Typical ellipse-shaped active zone containing two VGCC clusters, (ii) model-predicted maps of the overall release probability, and (iii) of the relative fractions of synchronous and asynchronous release modes.

(D) Results of a representative simulation. (i) Grey circles depict randomly assigned locations of vesicular release sites. Blue and red dots correspond to synchronous and asynchronous release events respectively, which occurred during a simulated train of 51 action potentials. (ii) To mimic the experimental data, locations of release events were blurred by adding a random value corresponding to the accuracy of SF-iGluSnFR event localisation (75 nm). (iii) Comparison of convex hull areas circumventing simulated locations of synchronous or asynchronous events. 
(E, F) Comparison of the model's output to the experimental results. The simulated data from $\mathrm{n}=100$ runs (boutons) were processed using the same analysis routine as for the experimental data set. (E) Model-predicted relationship between asynchronous release fraction and the overall vesicular release rate $n_{T}$ (similar to analysis in Fig. 2A). (F) Results of reshuffling analysis (similar to Fig. 3H, I).

(G) Distribution of distances to the centre of the active zone for simulated synchronous and asynchronous release events. In agreement with recent electron microscopy data ${ }^{4,12}$ the model predicts that locations of asynchronous release events are expected to be biased towards the centre of the active zone.

$* \mathrm{p}<0.05, * * * \mathrm{p}<0.001, \mathrm{NS} \mathrm{p}>0.2$, Mann-Whitney U test. 


\section{Supplementary Figures}
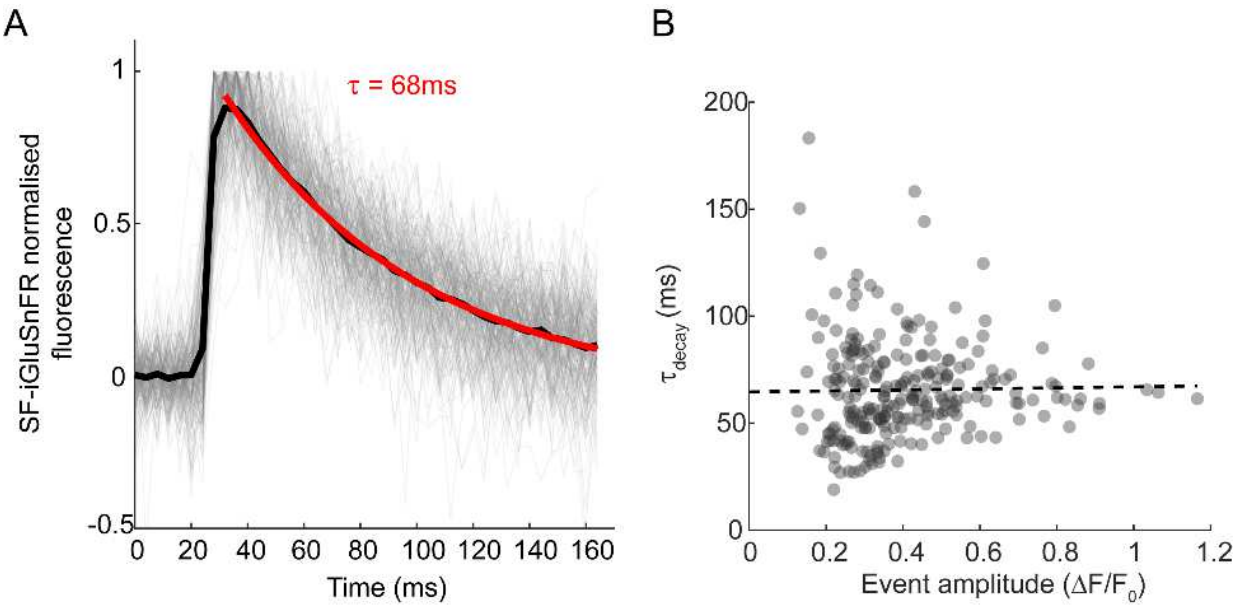

Fig. S1. Analysis of kinetics of evoked presynaptic SF-iGluSnFR events.

(A) Black trace, average of 244 individual action potential-evoked SF-iGluSnFR fluorescence transients (grey traces) aligned by their rising slopes $(n=37$ representative presynaptic boutons recorded in $\mathrm{N}=6$ wild type cells). Red trace, mono-exponential fit of the decay phase of the average trace $F(t)=e^{-t / \tau}$.

(B) The values of decay time constant $\tau$ estimated for individual SF-iGluSnFR events do not correlate with the event amplitude. Dashed line, linear regression. 
A
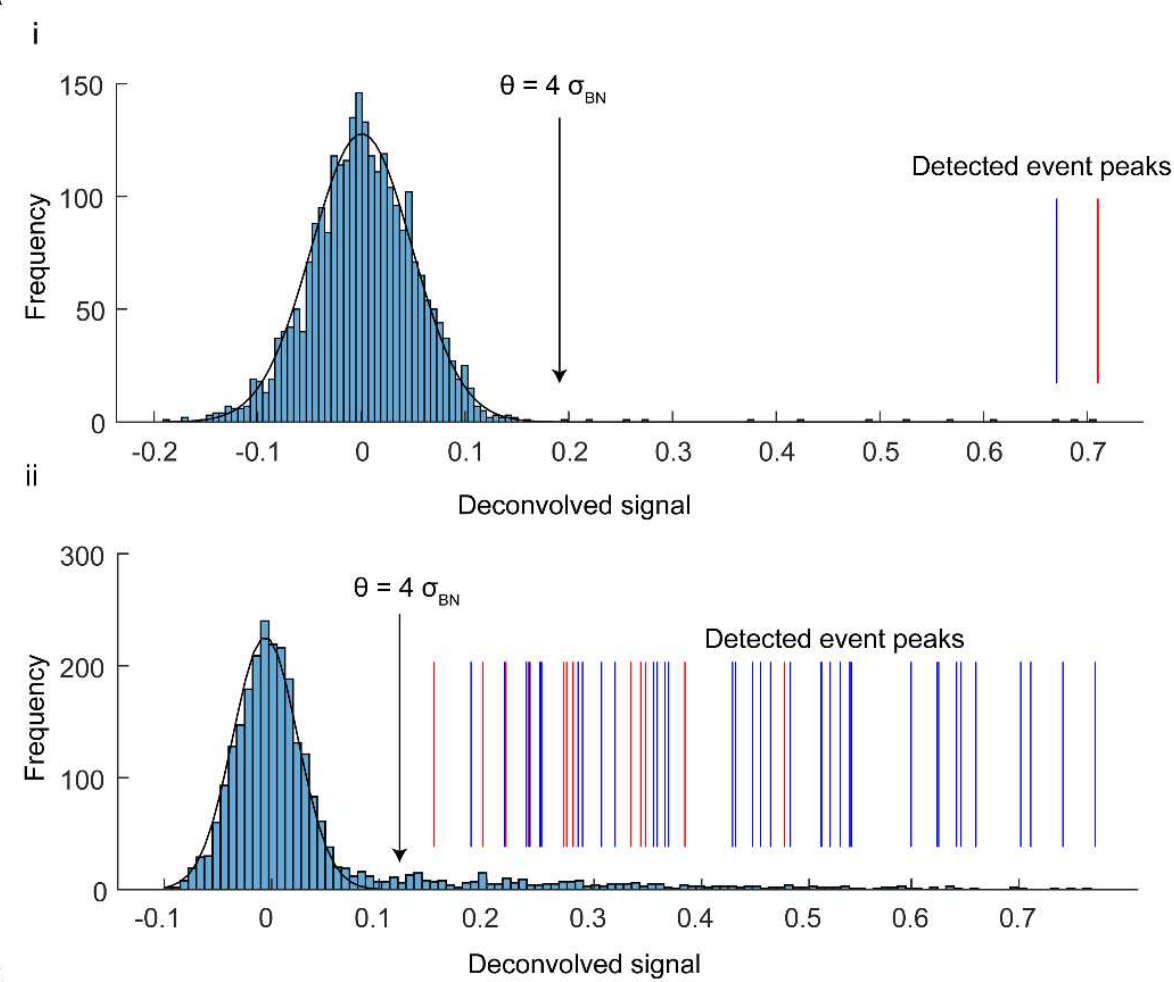

B

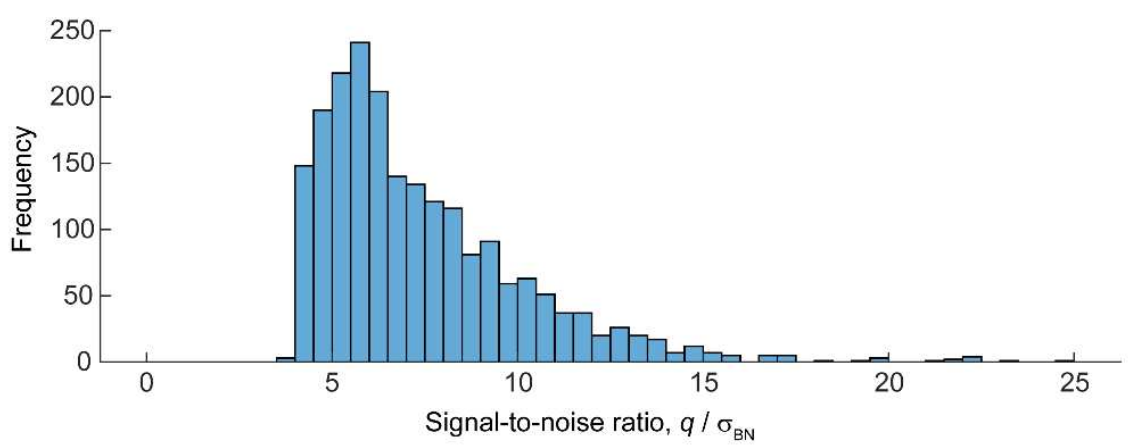

Fig. S2. Analysis of background noise and setting a threshold for automatic detection of quantal glutamate release events in single boutons.

(A) All-point histograms of the deconvolved traces from boutons 1 and 2 in Fig. 1. Black lines, histogram fits with a Gaussian function $\frac{A}{\sigma_{B N} \sqrt{2 \pi}} \exp \left(\frac{-x^{2}}{2 \sigma_{B N}^{2}}\right)$. The obtained standard deviation $\sigma_{B N}$, which characterises the baseline noise, was used to set-up the bouton-specific threshold for detection of quantal events: $\theta=4 \sigma_{B N}$ (vertical arrows). Vertical bars depict the amplitudes of SF-iGluSnFR synchronous (blue) and asynchronous (red) release events, determined as local maxima located above the threshold on the deconvolved trace in Fig. 1E.

(B) Distribution of signal-to-noise ratio across recorded synapses (pooled data from $\mathrm{N}=16$ wild type and $\mathrm{N}=11 \mathrm{Syt1}^{-/-}$neurons, total $\mathrm{n}=2,075$ boutons). Signal-to-noise ratio in each bouton was calculated as the ratio of SF-iGluSnFR signal amplitude corresponding to release of a single vesicle (1q) to the standard deviation of the background noise $\sigma_{B N}$. 
A

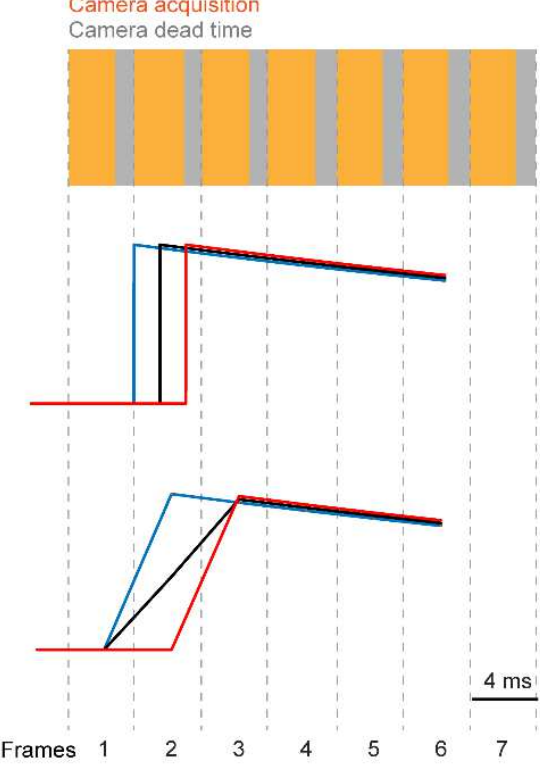

B

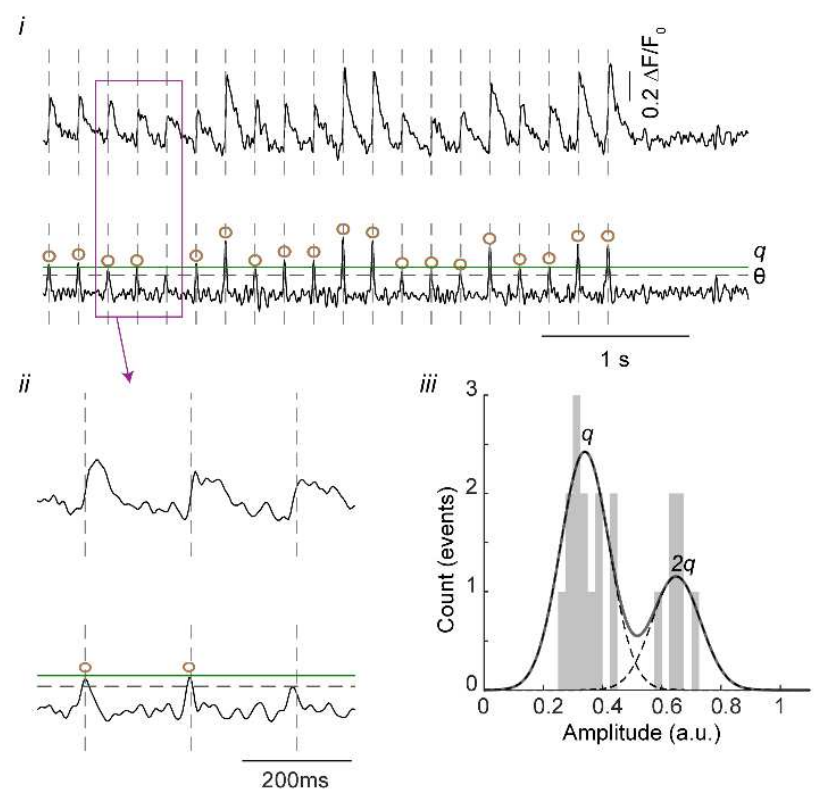

。

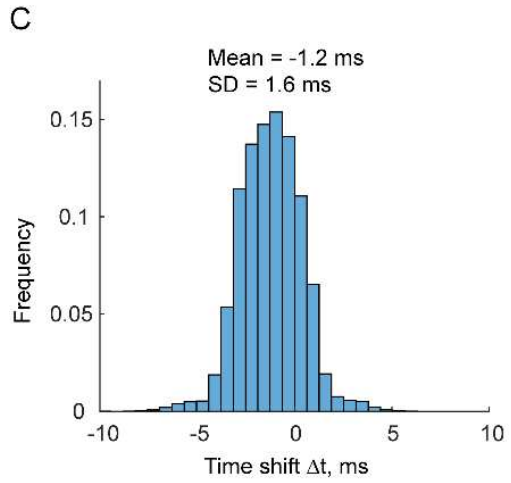

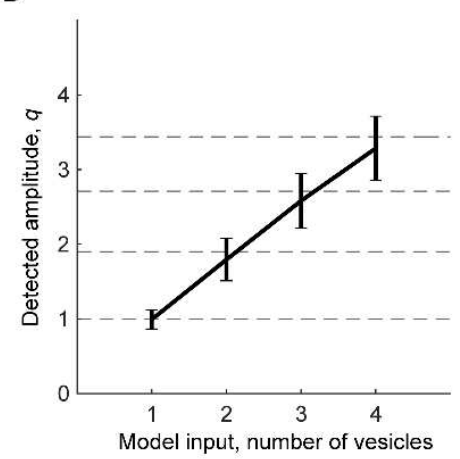

E

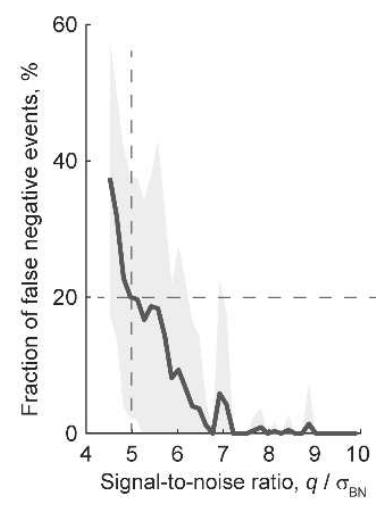

Fig. S3. Temporal resolution and sensitivity of quantal analysis.

(A) Illustration of the effect of a random jitter between the exact vesicular release times and the camera exposure cycle on the profile of SF-iGluSnFR responses. To ensure equal detection of fluorescence signals from all pixels within a selected ROI (typically $1412 \times 125$ ) the Prime95B camera was operated in the 'Pseudo Global Shutter' mode, when excitation light was only delivered during simultaneous exposure of all selected camera rows. As a result, photons were only collected during the first $2.8 \mathrm{~ms}$ (yellow rectangles) of the $4 \mathrm{~ms}$ frame cycle (vertical dashed lines). Middle traces illustrate 'ideal' SF-iGluSnFR signals that initiate at different times during the frame cycle. Bottom traces depict the shape of corresponding SFiGluSnFR signals as they would be acquired by the camera.

(B) Analysis of simulated SF-iGluSnFR responses. Each of 1,000 simulated traces consisted of 1,200 x 4 ms frames. Vesicular release events ( 20 per trace) were assumed to occur at $5 \mathrm{~Hz}$ (i.e. every $50^{\text {th }}$ frame). The exact timings of release events within the selected frames were random. The number of vesicles released during each event was drawn from a binomial distribution, assuming $m=5$ (the number of release ready vesicles) and $p_{v}=0.15$ (release probability of an individual vesicles). The amplitudes of SF-iGluSnFR responses 
corresponding to simultaneous release of $i$ vesicles were approximated as $\mu_{i}=\sum_{k=1}^{i} \lambda^{(k-1)} \mu_{1}$, where $\lambda=0.9$ is a factor (estimated experimentally) that accounts for a progressive saturation of SF-iGluSnFR during multi-vesicular release (see Methods). The time course of SFiGluSnFR responses was approximated as an instantaneous rise followed by an exponential decay with a characteristic time $\tau=68 \mathrm{~ms}$ (Fig. S1). The signal-to-noise ratio for each trace was randomly drawn from the experimentally determined distribution (Fig. S2B).

(i) Representative band-pass filtered (top) and deconvolved (bottom) simulated SF-iGluSnFR signals with signal-to-noise ratio $5.7 q / \sigma_{B N}$. As in the case of experimental traces (Fig. 1E), release events (brown circles) were identified as local maxima on the deconvolved trace located above the threshold $\theta=4 \sigma_{B N}$ (horizontal dashed line) corresponding to 4 standard deviations of the background noise.

(ii) Enlarged traces corresponding to the boxed area containing a false-negative (missed) event. (iii) Right, quantal analysis of the example artificial trace performed as detailed in Fig. 1E.

(C) Distribution of the time shifts $(\Delta t)$ between the determined and the actual times of simulated events. The results of simulations confirm that temporal resolution of our analysis is limited by the camera acquisition rate ( $4 \mathrm{~ms} /$ frame). The detected event times were systematically shifted to the left by approximately $1.25 \mathrm{~ms}$. This was due to a combined effect of noise, bandpass filtering and the inherent random jitter between the timings of release events and the camera exposure cycle (A). Considering the intrinsic $2-6 \mathrm{~ms}$ delay in the onset of synchronous release caused by the finite speed of action potential propagation and variable distances between synapses and cell soma ${ }^{19}$ we did not perform correction for this small systematic error.

(D) Relationship between model input (number of vesicles) and model output (detected amplitude of deconvolved SF-iGluSnFR signal in units of $q$ ) verifying the robustness of the amplitude histogram fitting approach. Horizontal dashed lines depict model-predicted SFiGluSnFR amplitude values ( $\mu_{i}=\sum_{k=1}^{i} \lambda^{(k-1)} \mu_{1}$, see Methods).

(E) Dependency of false-negative event fraction on the signal-to-noise ratio. Based on this simulation, boutons with signal-to-noise ratio below $5 q / \sigma_{B N}$ (vertical dashed line) were excluded from analysis, thus restricting the fraction of false-negative events to $\sim 20 \%$ (horizontal dashed line). 
A

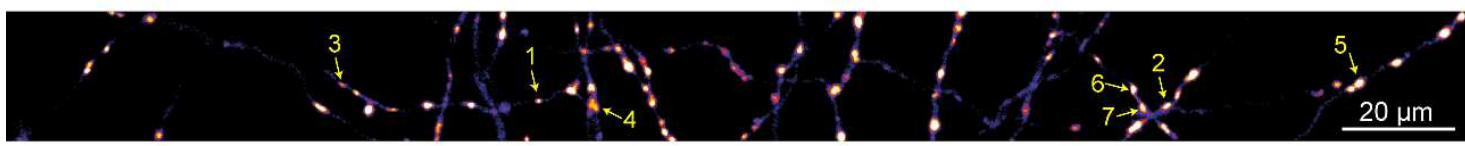

B
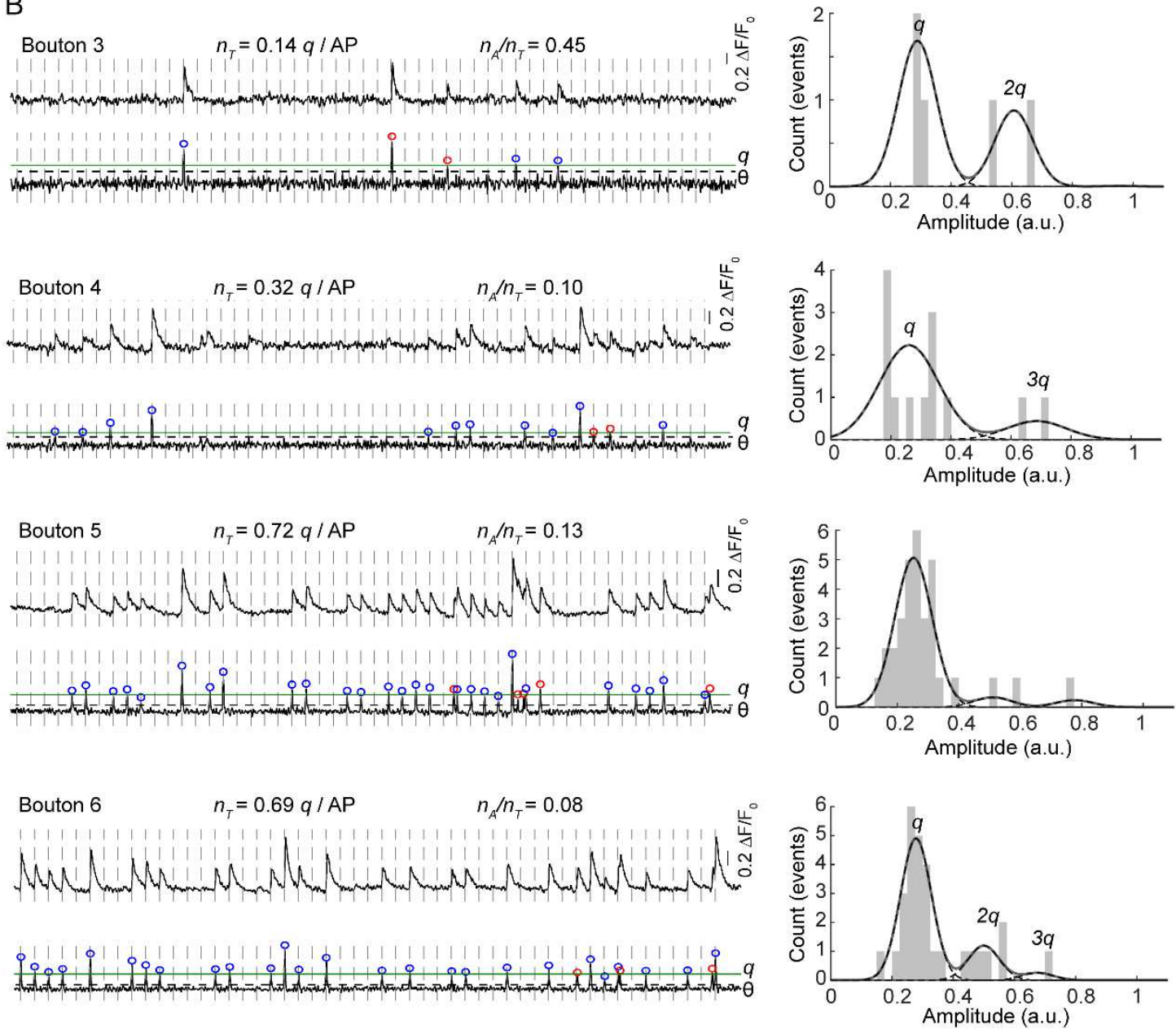

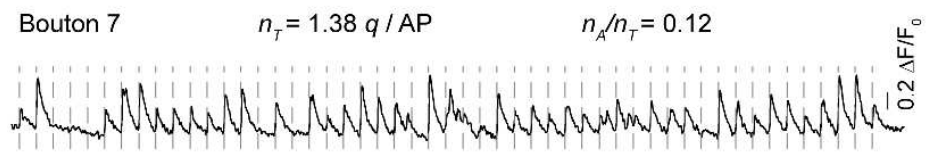
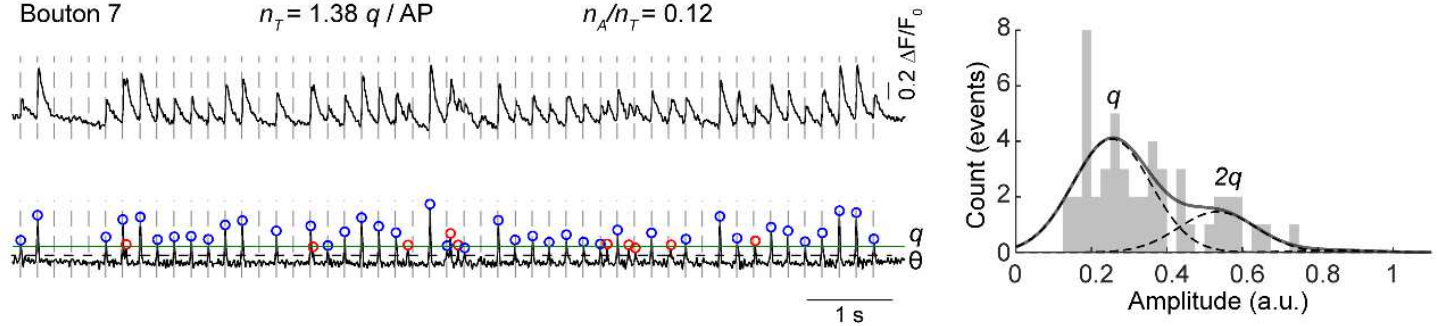

\section{Fig. S4. Additional examples of quantal analysis in individual presynaptic boutons (the same wild type neuron as in Fig. 1).}

(A) Heat map of glutamate release sites (maximal projection of the band pass-filtered SFiGluSnFR image stack, same image as in Fig. 1D). Yellow arrows depict positions of example boutons. (B) Quantal analysis of SF-iGluSnFR responses at Boutons 3 to 7 (see Fig.1E for analysis of boutons 1 and 2). Left, band-pass filtered (top traces) and deconvolved (bottom traces) SF-iGluSnFR signals. Vertical dashed lines depict the timings of somatic action potentials. Horizontal black dashed lines - detection thresholds $(\theta)$ for vesicular release events, corresponding to 4 standard deviations of the background noise. Horizontal green lines 
- amplitudes of SF-iGluSnFR signals corresponding to release of a single vesicle (single quanta, $q$ ) determined by fitting positions of peaks on the amplitude histograms (right). Blue and red circles on the deconvolved traces depict synchronous and asynchronous release events respectively. Comparison of quantal responses at boutons 6 and 7 illustrates that SF-iGluSnFR signals in neighbouring boutons are not cross-contaminated by a possible glutamate spillover. 
A

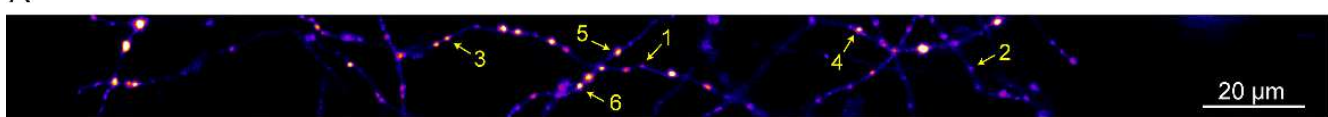

B
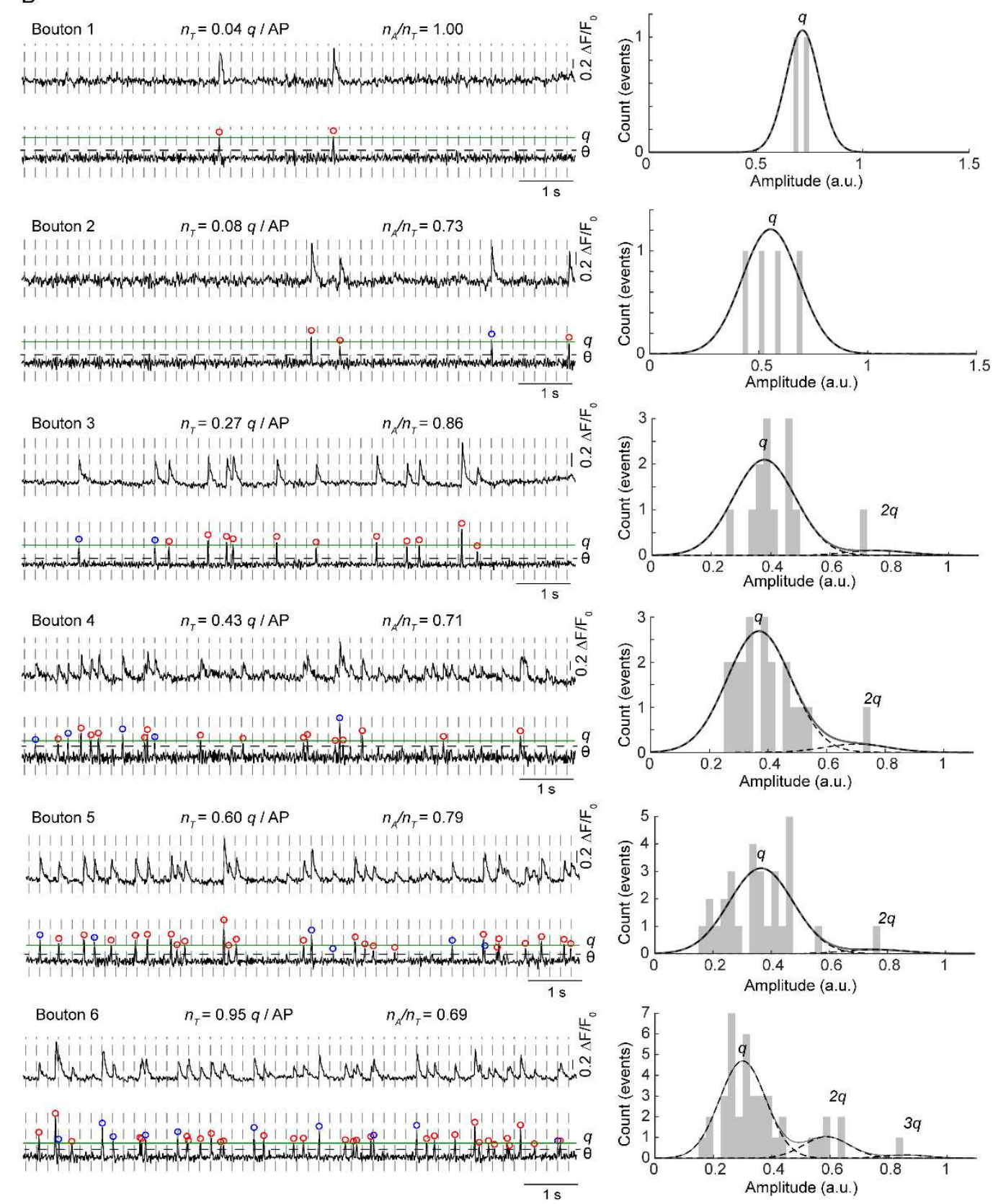

Fig. S5. Examples of quantal analysis in individual presynaptic boutons in a representative $\mathrm{Syt1}^{-/-}$neuron.

(A) Heat map of glutamate release sites (maximal projection of the band pass-filtered SFiGluSnFR image stack) across the axonal arbour of a Syt $1^{-/}$neuron. Yellow arrows depict positions of six example boutons.

(B) Analysis of SF-iGluSnFR responses in the selected boutons. Left, band-pass filtered (top traces) and deconvolved (bottom traces) SF-iGluSnFR signals. Vertical dashed lines depict the timings of somatic action potentials. Horizontal black dashed lines - detection thresholds $(\theta)$ for vesicular release events, corresponding to 4 standard deviations of the background noise. 
Horizontal green lines - amplitudes of SF-iGluSnFR signals corresponding to release of a single vesicle $(q)$ determined by fitting positions of peaks on the amplitude histograms (right). Blue and red circles on the deconvolved traces depict synchronous and asynchronous release events respectively. 


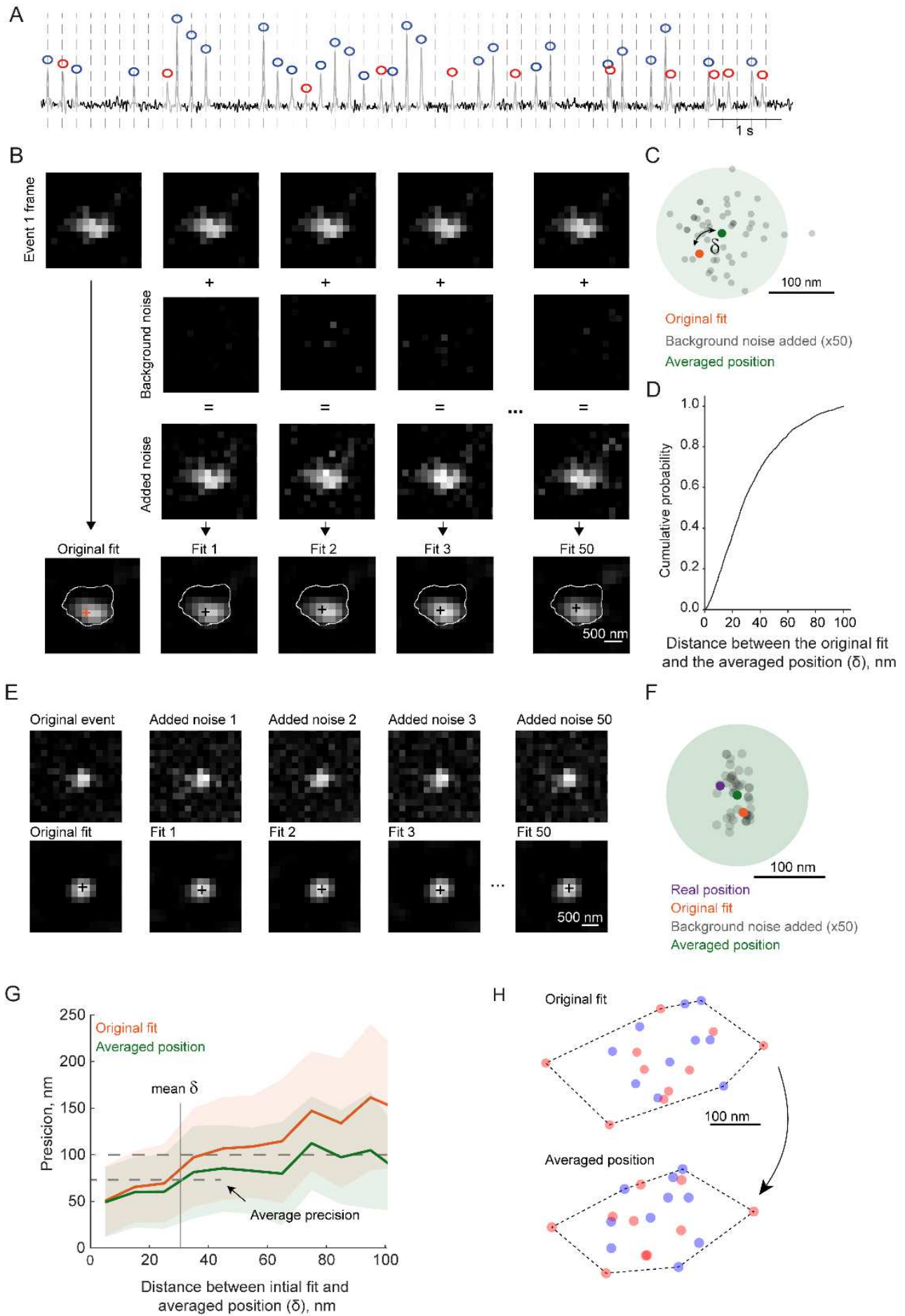

Fig. S6. Estimation and improvement of vesicular release sites localisation precision using noise analysis.

(A) Generation of 'background noise' image stack (1000 images). Each background noise image was obtained by averaging 3 randomly selected frames from the deconvolved SFiGluSnFR image stack, which were separated from the nearest release event by at least 15 frames (depicted in black on the representative trace, the same bouton as in Fig. 3A-D).

(B, C) Estimation of vesicular release localisation precision (detailed illustration for Event 1 from Fig. 3A-D). (B) For each event, an 'added noise' image stack was generated (50x), where each image was a sum of the original 'event image' and a randomly selected image from the 
'background noise' stack. We next run the sub-pixel localisation analysis for each image in the 'added noise' stack (grey dots in C), calculated their average position (green dot) and the distance $(\delta)$ between location of the 'original' fit (obtained using the initial 'event image', orange dot) and the average position from the 'added noise' stack. Events with $\delta$ above the $100 \mathrm{~nm}$ threshold were excluded from analysis.

(D) Cumulative distribution of $\delta$ values for 2,592 fitted events from $\mathrm{n}=106$ wild type boutons, $\delta_{\text {mean }}=32.4 \pm 23.0 \mathrm{~nm}$ (mean \pm standard deviation).

(E to $\mathbf{G})$ Testing if $\delta$ represents a realistic estimate of the localisation accuracy by performing localisation analysis on artificial computer-simulated images (see Methods). (E, F) A representative simulation. Purple point in (F) depicts the real position of the simulated event. (G) Dependency of the localisation precision (defined as the distance to the real position either from the 'original fit' or from the 'averaged position') on $\delta(\mathrm{N}=1500$ simulations, shaded areas depict standard deviation). The simulations reveal that the averaged position determined from the 'added noise' stack provides a more reliable estimate for the location of release site. Therefore, we used this improved averaged fit in the analysis of the relative distributions of synchronous and asynchronous release events. Considering that $\delta_{\text {mean }}=32.4$, the average accuracy in the localisation of exocytosis events in our experimetns was $\sim 75 \mathrm{~nm}$ (in the range between $50-100 \mathrm{~nm}$ ).

(H) Comparison of the original and the improved localisation maps for the bouton shown in Fig. 3A-D. 

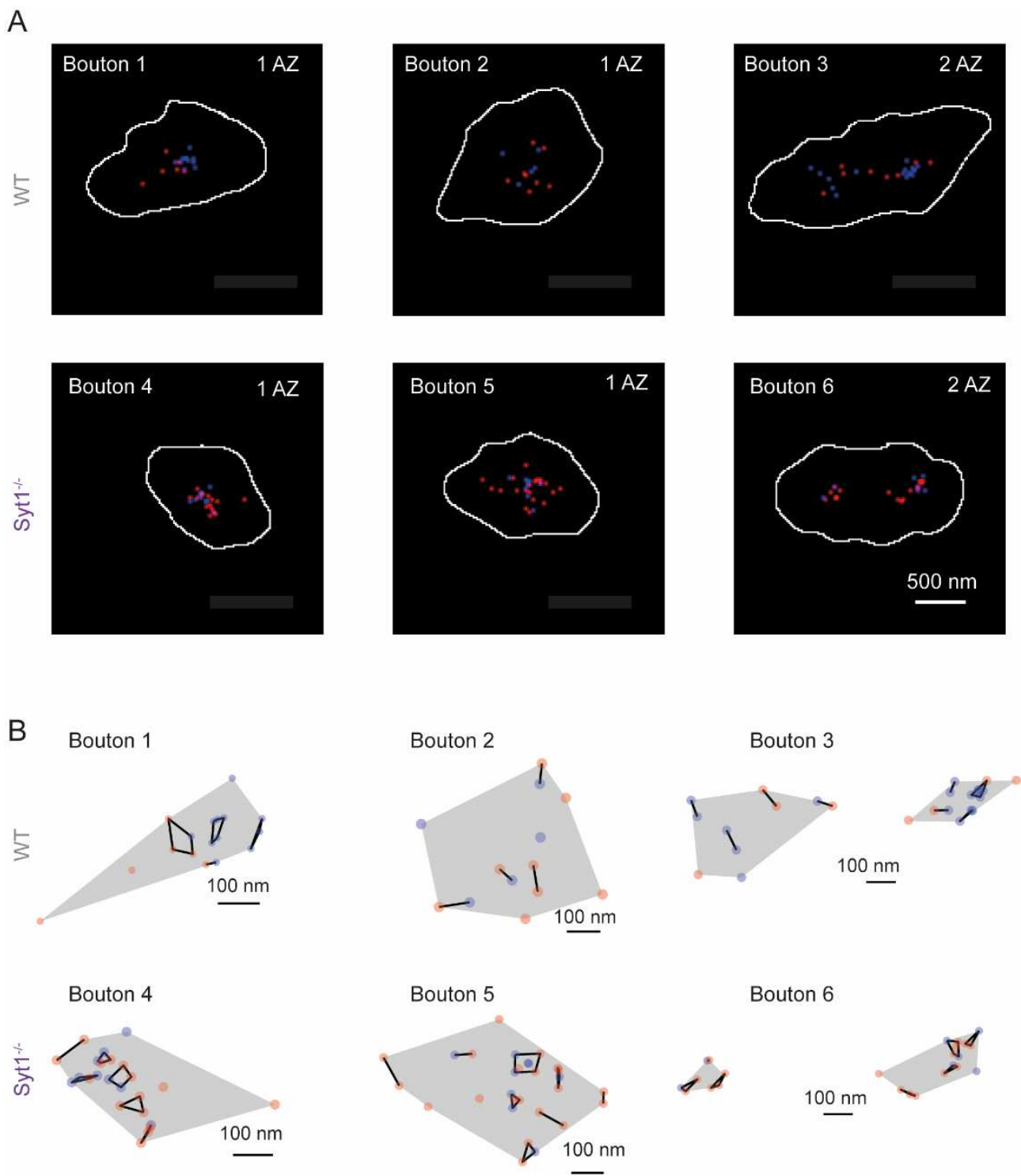

Fig. S7. Additional examples of spatial distributions of synchronous and asynchronous exocytosis sites.

(A) Composite images showing relative locations of synchronous (blue) and asynchronous (red) release events in several representative wild type boutons (WT, 1-3) and Syt1/- boutons (4 - 6), see also Fig. 3D.

(B) Hierarchical cluster analysis of vesicular exocytosis sites in the same boutons as in (A). Putative active zones (grey shaded areas) were identified using a clustering diameter threshold of $700 \mathrm{~nm}$. Boutons with 2 active zones (e.g. boutons 3 and 6) were excluded from further analysis. To compare the relative locations of synchronous and asynchronous release sites, the positions of vesicular release events were grouped into clusters using a clustering diameter threshold of $100 \mathrm{~nm}$ (see Fig. 3F and the main text for details). 
Movie S1. Identification of active presynaptic boutons.

Animated version of Fig. 1B-D.

Movie S2. Sub-pixel localisation of synchronous and asynchronous release events.

Animated version of Fig. 3C. Scale bar $500 \mathrm{~nm}$. 


\section{Supplementary Files}

This is a list of supplementary files associated with this preprint. Click to download.

- SupplementaryMovie1.mp4

- SupplementaryMovie2.mp4 